\title{
Glucose intolerance in aging is mediated by the Gpcpd1-GPC metabolic axis
}

\author{
Domagoj Cikes $^{1 *}$, Michael Leutner ${ }^{2}$, Shane Cronin ${ }^{1}$, Maria Novatchkova ${ }^{1}$, Lorenz Pfleger ${ }^{2}$, \\ Radka Klepochová2 ${ }^{2}$ Eric Rullman ${ }^{4,5}$, Thomas Gustafsson ${ }^{4}$, Astrid Hagelkruys ${ }^{1}$, Michael \\ Krebs $^{2}$, Alexandra Kautzky-Willer ${ }^{2}$, Martin Krssak ${ }^{2}$, Michael Orthofer ${ }^{1}$, Josef M. \\ Penninger $^{1,3^{*}}$
}

${ }^{1}$ IMBA, Institute of Molecular Biotechnology of the Austrian Academy of Sciences, Vienna, 1030, Austria

${ }^{2}$ Division of Endocrinology and Metabolism Department of Internal Medicine III, Medical University of Vienna, Vienna, 1090, Austria

${ }^{3}$ Department of Medical Genetics, Life Sciences Institute, University of British Columbia, Vancouver; Canada

${ }^{4}$ Division of Clinical Physiology, Department of Laboratory Medicine, Karolinska Institutet, and Unit of Clinical Physiology, Karolinska University Hospital, Stockholm; Sweden

${ }^{5}$ Cardiovascular Theme, Karolinska Institutet, Karolinska University Hospital Huddinge, Stockholm; Sweden

*Correspondence

domagoj.cikes@imba.oeaw.ac.at (D.C.), josef.penninger@ubc.ca (J.M.P) 


\begin{abstract}
Skeletal muscle plays a central role in the regulation of systemic metabolism during lifespan. With aging, muscle mediated metabolic homeostasis is perturbed, contributing to the onset of multiple chronic diseases. Our knowledge on the mechanisms responsible for this agerelated perturbation is limited, as it is difficult to distinguish between correlation and causality of molecular changes in muscle aging. Glycerophosphocholine phosphodiesterase 1 (GPCPD1) is a highly abundant muscle enzyme responsible for the hydrolysis of the lipid glycerophosphocholine (GPC). The physiological function of GPCPD1 remained largely unknown. Here, we report that the GPCPD1-GPC metabolic pathway is dramatically perturbed in the aged muscle. Muscle-specific inactivation of Gpcpd1 resulted in severely affected glucose metabolism, without affecting muscle development. This pathology was muscle specific and did not occur in white fat-, brown fat- and liver-specific Gpcpd1 deficient mice. Moreover, in the muscle specific mutant mice, glucose intolerance was markedly accelerated under high sugar and high fat diet. Mechanistically, Gpcpd1 deficiency results in accumulation of GPC, without any other significant changes in the global lipidome. This causes an "aged-like" transcriptomic signature in young Gpcpd1 deficient muscles, changes in myofiber osmolarity, and impaired insulin signaling. Finally, we report that GPC levels are markedly perturbed in muscles from both aged humans and patients with Type 2 diabetes. These results identify the GPCPD1-GPC metabolic pathway as critical to muscle aging and age-associated glucose intolerance.
\end{abstract}

Keywords: Aging; Muscle; Glycerophosphocholine phosphodiesterase 1; Glycerophosphocholine; Glucose intolerance; Metabolic dysfunction; Insulin signaling; 


\section{Introduction}

Skeletal muscle is the biggest organ in the body, essential for mobility, health-span and lifespan (Cruz-Jentoft and Sayer, 2019; Yanagi et al., 2021; Yang et al., 2019). Apart from mobility, muscle plays a crucial role in systemic metabolism (Baskin et al., 2015). Critical to this regulatory role is the control of glucose metabolism, with muscle metabolizing up to $80 \%$ of ingested glucose (DeFronzo et al., 1981; Jue et al., 1989). Aged muscles exhibit multiple molecular and metabolic perturbations, that can potentially affect muscle, and consequently whole-body metabolism during the lifespan (Demontis et al., 2013a). With muscle ageing, glucose utilization is significantly impaired, leading to systemic perturbations of glucose metabolism (Chia et al., 2018; Kern et al., 1992). This systemic metabolic dysfunction contributes to onset of many other chronic diseases (Cowie et al., 2009).

Glycerophosphodiester phosphodiesterase 1 (Gpcpd1; Gdpd6; Edi3) is a member of a large family of glycerophosphodiester phosphodiesterases, highly conserved enzymes found in bacteria, protozoa, and mammals (Corda et al., 2014). Mammalian glycerophosphodiesterases exist in multiple isoforms (seven in humans) with a high degree of tissue and substrate specificity (Corda et al., 2014). Gpcpd1 is a $76.6 \mathrm{kD}$ protein that hydrolyzes lipid glycerophosphocholine (GPC), yielding choline and glycerol-3-phosphate. Unlike other phosphodiesterases, Gpcpd1 does not contain a transmembrane region and is localized in the cytoplasm. An early study reported that Gpcpd1 expression is enriched in heart and skeletal muscle (Okazaki et al., 2010). The same study also reported that overexpression of a truncated version of Gpcpd1 in the muscles resulted in muscle atrophy, suggesting that Gpcpd1 regulates muscle differentiation (Okazaki et al., 2010). High expression of Gpcpd1 was found in metastatic endometrial cancers, while inhibition of Gpcpd1 in breast cancer cells inhibited migration and invasion (Stewart et al., 2012). Apart from these studies, the physiological function of Gpcpd1 remains largely unknown, and an in vivo loss-of-function analysis has never been reported.

A recent untargeted metabolomic profiling of skeletal muscle from old mice indicated glycerophosphocholine (GPC), the Gpcpd1 substrate, as a significantly elevated metabolite 
in aged mouse muscles (Houtkooper et al., 2011). Moreover, a single nucleotide polymorphism in proximity to human GPCPD1 locus was found to be associated with longevity (Pilling et al., 2016) Therefore, we set out to investigate if there was a direct link between the Gpcpd1-GPC metabolic pathway and aging-related health decline.

\section{MATERIALS AND METHODS}

\section{Animal care}

All experimental protocols were approved by the institutional Animal Ethics Committee in accordance with the Austrian legal guidelines on Animal Care. All mice were on a C57BL/6J background (in-house colony). MckCre, Ap2Cre, Ucp1Cre, AlbCre mice were purchased from the Jackson Laboratory (Bar Harbor, US, stock number 006405, 005069, 024670, 003574). The mice were maintained on a 12:12 hour light-dark cycle (lights on 08:00-20:00) while housed in ventilated cages at an ambient temperature of $25^{\circ} \mathrm{C}$. Mice were fed ad libitum standard chow diet (17\% kcal from fat; Envigo, GmbH), high-fat diet (HFD; 60\% kcal from fat; Envigo, $\mathrm{GmbH}$ ), or high fructose diet (30\% fructose in drinking water; \#F0127 Sigma Aldrich $\mathrm{Gmbh}$ ) starting from 8 weeks of age.

\section{Human biopsies}

All human experiments were approved by the regional ethical review board in Stockholm (2014/516-31/2 and 2010/786-31/3) and complied with the Declaration of Helsinki. Oral and written informed consent were obtained from all subjects prior to participation in the study. 8 healthy young adults (age 21-29) and 8 middle-aged (age 45-62) subjects were recruited. The subjects did not use any medications and were nonsmokers. Biopsies of the quadriceps skeletal muscle (vastus lateralis) were obtained under local anesthesia using the Bergström percutaneous needle biopsy technique (Bergström and Hultman, 1967). The biopsies were immediately frozen in isopentane, cooled in liquid nitrogen, and stored at $-80^{\circ} \mathrm{C}$ until further analysis. 


\section{Determination of muscle glycerophosphocholine in Type 2 diabetes patients}

This prospective clinical study was performed at the Department of Internal Medicine 3, Division of Endocrinology and Metabolism at the Medical University of Vienna between June 2021 and September 2021. Patients were recruited at the diabetes outpatient department of the Medical University of Vienna and enrolled for the study after giving written informed consent. Routine laboratory measurements were analyzed at the certified Department of Medical and Chemical Laboratory Diagnostics (http://www.kimcl.at/) of the Medical University of Vienna. In the present study, we included patients with diagnosed diabetes mellitus type 2 and patients without diabetes mellitus aged between 50 and 65 years. Exclusion criteria were infectious diseases such as hepatitis B or C and human immunodeficiency virus (HIV). We performed a detailed metabolic characterization, including measurements of lipid parameters, glucose metabolism (e.g. HbA1c, fasting glucose levels), routine laboratory analyses and anthropometric measurements. In vivo magnetic resonance spectroscopy (MRS) of vastus lateralis muscle was performed as previously described (Beiglböck et al., 2020; Valkovič et al., 2013). Laboratory analyses and MRS measurements were performed after a 12-hour fasting period. The study was approved by the ethics board of the Medical University of Vienna.

\section{Cross tissue Gpcpd1 expression analysis}

The data on Gpcpd1 mRNA expression analysis in several tissues were derived from the gene expression profiling atlas GSE10246 (Lattin et al., 2008) and visualized using the BioGPS portal (Wu et al., 2016).

\section{Generation of Gpcpd1 conditional and tissue specific mutant mice}

Gpcpd1 conditional knockout mice were derived from targeted ES cells obtained from EUCOMM (European Conditional Mouse Mutagenesis Program). Exons 9 and exon 10 of the Gpcpd1 gene were flanked by loxP sites. Cre mediated deletion of the floxed region creates a frameshift and truncated protein. Upon confirmation of correct targeting by Southern blotting, the ES cell clone $\mathrm{G} 8(\mathrm{C} 57 \mathrm{BI} / 6 \mathrm{~N})$ was injected into $\mathrm{C} 57 \mathrm{BL} / 6 \mathrm{~J}-\mathrm{Tyr}^{\mathrm{c}-2 \mathrm{~J}} / \mathrm{J}$ blastocysts and offspring chimeric mice were crossed to $\mathrm{C} 57 \mathrm{BL} / 6 \mathrm{~J}$ mice. Following germline transmission, targeted mice were crossed to transgenic mice expressing FLPe recombinase leading to 
excision of the NEO cassette. Offspring mice were backcrossed onto a C57BI/6J genetic background. Following primers were used to identify the floxed allele:

Forward - GTGCAGGGAACTCAACAACG

Reverse - AGTGATGACAAAGAGGCCAAAAAG

Subsequently, MckCre, Ap2Cre, Ucp1Cre, and AlbCre mutant animals were crossed to the Gpcpd1 $1^{\text {flox/flox }}$ mice to generate MckCre-Gpcpd1, Ap2Cre-Gpcpd1, Ucp1Cre-Gpcpd1, and AlbCre-Gpcpd1 mutant mice.

\section{Quantitative real-time PCR (RT-qPCR)}

After animal sacrifice, tissues were extracted, separated into regions and flash frozen in liquid nitrogen. Subsequently, mRNA was isolated using the RNeasy Lipid Tissue Mini Kit (Qiagen, $\mathrm{GmbH}$ ). The RNA concentrations were estimated by measuring the absorbance at $260 \mathrm{~nm}$ using Nanodrop (Thermofisher, $\mathrm{GmbH}$ ). cDNA synthesis was performed using the iScript Advanced cDNA Synthesis Kit for RT-qPCR (Bio-Rad, GmbH) following manufacturer's recommendations. CDNA was diluted in DNase-free water (1:10) before quantification by real-time PCR. mRNA transcript levels were measured in triplicate samples per animal using CFX96 touch real-time PCR (Bio-Rad GmbH). Detection of the PCR products was achieved with SYBR Green (Bio-Rad, GmbH). At the end of each run, melting curve analyses were performed, and representative samples of each experimental group were run on agarose gels to ensure the specificity of amplification. Gene expression was normalized to the expression level of $18 \mathrm{~S}$ ribosomal rRNA as the reference gene. The following primers were used:

Murine Gpcpd1:

Forward 5'-GTGGTGCAGGGAACTCAACAACG - 3'

Reverse 5'- TGAGGTCATGATACACCACGGGC - 3'

Murine 18SrRNA:

Forward 5'- GGCCGTTCTTAGTTGGTGGAGCG -3'

Reverse 5'- CTGAACGCCACTTGTCCCTC - 3'

Human Gpcpd1:

Forward 5'- GCATCTGTGGTGCTAGGTGA - 3'

Reverse 5'- TGCCTTGTGAAAAACATGCAG - 3'

Human 18SrRNA: 
Forward 5'- GGCCCTGTAATTGGAATGAGTC -3'

Reverse 5'- CCAAGATCCAACTACGAGCTT - 3'

\section{Glucose tolerance test (GTT), insulin tolerance test (ITT) and insulin measurements}

For glucose tolerance tests (GTT), mice were fasted for $16 \mathrm{~h}$ and a D-glucose solution was administered by oral gavage at a dose of $2 \mathrm{~g} / \mathrm{kg}$ (chow diet), or $1 \mathrm{~g} / \mathrm{kg}$ (high sugar, high fat diet). Blood samples were collected from the tail vein and glucose was measured using a glucometer (Roche, Accu-Chek Performa). For analysis of insulin levels, tail vein blood samples were added to a $\mathrm{NaCl} / \mathrm{EDTA}$ solution to avoid blood clotting and plasma insulin concentrations were determined using the Alpco Mouse Ultrasensitive Insulin ELISA (80INSMSU-E10). For insulin tolerance tests (ITT), mice were fasted for $6 \mathrm{~h}$ and insulin solution (0.75 IU insulin $/ \mathrm{kg}$ ) administered i.p., followed by blood glucose measurements.

\section{Determination of muscle glycogen levels}

Skeletal muscle tissue (quadriceps) was surgically removed, and flash frozen in liquid nitrogen. The tissue was crushed on dry ice, weighed, and further processed according to the manufacturers' instructions (Sigma Aldrich; \#MAK016). Tissue glycogen levels were determined based on the formulation derived from the standard curve and normalized to the tissue weight.

\section{Muscle RNA purification}

Total RNA was extracted from skeletal muscles using the Qiagen miRNeasy Mini kit (cat\# 217004, Qiagen, GmbH) per the manufacturer's instructions. In brief, samples were homogenized in QIAzol lysis reagent using a rotor stator homogenizer, and then centrifuged after chloroform addition at 12,000? RNA was purified from the aqueous phase using the spin column provided in the kit. DNA was digested on-column per the manufacturer's instructions. RNA concentration was measured using the Nanodrop and RNA integrity was measured with an Agilent 2200 Tapestation instrument (cat\#5067-5576 and cat\#5067-5577, Agilent, Santa Clara, CA). All samples had RIN values greater than 8. 


\section{Quantseq analysis and sequencing}

Libraries were prepared using the QuantSeq 3'mRNA-Seq Library Prep Kit-FWD (cat \#15, Lexogen, Vienna, Austria) using 1? $\mu \mathrm{g}$ of RNA per library, following manufacturer's instructions.

11? cycles of library amplification were performed with indices from the first two columns of the i7 Index Plate for QuantSeq/SENSE with Illumina adapters 7001-7096 (cat \#044, Lexogen). Libraries were eluted in 22? $\mu \mathrm{L}$ of the Elution Buffer, and double stranded DNA concentration was quantified by the KAPA Library Quantification Kit. The molar concentration of CDNA molecules was calculated from the double stranded DNA concentration and the region average size (determined by analyzing each sample on an Agilent 2200 Tapestation instrument (cat\#5067-5584 and cat\#5067-5585, Agilent, Santa Clara, CA)). Aliquots containing an equal number of nmoles of cDNA molecules from each library were pooled to a concentration of 10 ? $\mathrm{nM}$ of cDNA. The final pool was purified once more (to remove any free primers to prevent index-hopping) by adding $0.9 x$ volumes of PB and proceeding from Step 30 onwards in the QuantSeq User Guide protocol. The library was eluted in 22? $\mu \mathrm{L}$ of the kit's Elution Buffer. The pooled libraries were sequenced using an Illumina HiSeq4000 instrument (Illumina, San Diego, CA).

\section{Transcript coverage}

RNA-seq reads were trimmed using BBDuk v38.06 (ref=polyA.fa.gz,truseq.fa.gz k=13 ktrim=r useshortkmers $=t$ mink= $=5$ trim $=r$ trimq $=10$ minlength $=20$ ) and reads mapping to abundant sequences included in the iGenomes Ensembl GRCm38 bundle (mouse rDNA, mouse mitochondrial chromosome, phiX174 genome, adapter) were removed using bowtie2 v2.3.4.1 alignment. Remaining reads were analyzed using genome and gene annotation for the $\mathrm{GRCm} 38 / \mathrm{mm} 10$ assembly obtained from Mus musculus Ensembl release 94 . Reads were aligned to the genome using star v2.6.0 c and reads in genes were counted with featureCounts (subread v1.6.2) using strand-specific read counting for QuantSeq experiments (-s 1). Differential gene expression analysis on raw counts and variancestabilized transformation of count data for heatmap visualization were performed using DESeq2 v1.18.1. Functional annotation overrepresentation analysis of differentially expressed genes was conducted using clusterprofiler v3.6.0 in R v3.4.1. 


\section{Western blot analysis and immunofluorescence}

A D-glucose solution was administered by oral gavage and mice were sacrificed after 15 minutes. Muscle tissue (quadriceps) was immediately surgically removed, and flash frozen in liquid nitrogen. Tissues were further homogenized in RIPA buffer (Sigma; R0278) containing Halt protease and phosphatase inhibitor cocktail (Thermo Scientific; 78440). Protein levels were determined using the Bradford assay kit (Pierce, $\mathrm{GmbH}$ ) and lysates containing equal amounts of protein were subjected to SDS-PAGE, further transferred to nitrocellulose membranes. Western blotting was carried out using standard protocols. Blots were blocked for 1 hour with 5\% BSA (Sigma Aldrich; \#820204) in TBST (1× TBS and 0.1\% Tween-20) and were then incubated overnight at $4^{\circ} \mathrm{C}$ with primary antibodies diluted in $5 \%$ BSA in TBST (1:250 dilution). Blots were washed three times in TBST for $15 \mathrm{~min}$, then incubated with HRP-conjugated secondary antibodies diluted in 5\% BSA in TBST (1:5000 dilution) for 45 min at room temperature, washed three times in TBST for $15 \mathrm{~min}$ and visualized using enhanced chemiluminescence (ECL Plus, Pierce, 1896327). The following primary antibodies were used: anti-phospho-Insulin Receptor $\beta$ (Tyr1146) (\# 3021 CST, DE, 1:250), anti-total Insulin Receptor $\beta$ (\# 3025 CST, DE, 1:250), anti-phospho-Akt (Ser473) (\# 4060 CST, DE, 1:250), antitotal Akt (\#4685, CS, DE 1:250). Secondary antibodies were anti-rabbit IgG HRP (CST, DE, \#7074).

For immunocytochemistry, quadriceps muscles were harvested and fixed in PFA (4\%) for $72 \mathrm{~h}$ and cryoprotected by further immersing in 30\% sucrose solution for another $72 \mathrm{hr}$. After embedding in OCT, sections were cut and stained using standard immunohistochemistry using the following antibodies: anti-Dystrophin (\#ab15277, Abcam, UK1:150).

\section{Lipidomics}

Quadriceps muscles were isolated from 8-week-old mice and snap frozen in liquid nitrogen. Muscle tissue was homogenized using a Precellys 24 tissue homogenizer (Precellys CK14 lysing kit, Bertin). Per mg tissue, $3 \mu \mathrm{L}$ of methanol were added. $20 \mu \mathrm{L}$ of the homogenized tissue sample was transferred into a glass vial, into which $10 \mu \mathrm{L}$ internal standard solution 
(SPLASH ${ }^{\circledast}$ Lipidomix $^{\circledast}$, Avanti Polar Lipids) and $120 \mu \mathrm{L}$ methanol were added. After vortexing, $500 \mu \mathrm{L}$ Methyl-tert-butyl ether (MTBE) were added and incubated in a shaker for $10 \mathrm{~min}$ at room temperature. Phase separation was performed by adding $145 \mu \mathrm{L}$ MS-grade water. After 10 min of incubation at room temperature, samples were centrifuged at $1000 \mathrm{xg}$ for $10 \mathrm{~min}$. An aliquot of $450 \mu \mathrm{L}$ of the upper phase (organic) was collected and dried in a vacuum concentrator. The samples were reconstituted in $200 \mu \mathrm{L}$ methanol and used for LCMS analysis. The LC-MS analysis was performed using a Vanquish UHPLC system (Thermo Fisher Scientific) combined with an Orbitrap Fusion ${ }^{\mathrm{TM}}$ Lumos $^{\mathrm{TM}}$ Tribrid $^{\mathrm{TM}}$ mass spectrometer (Thermo Fisher Scientific). Lipid separation was performed by reversed phase chromatography employing an Accucore C18, $2.6 \mu \mathrm{m}, 150 \times 2$ mm (Thermo Fisher Scientific) analytical column at a column temperature of $35^{\circ} \mathrm{C}$. As mobile phase $\mathrm{A}$ we used an acetonitrile/water (50/50, v/v) solution containing $10 \mathrm{mM}$ ammonium formate and $0.1 \%$ formic acid. Mobile phase B consisted of acetonitrile/isopropanol/water (10/88/2, v/v/v) containing $10 \mathrm{mM}$ ammonium formate and $0.1 \%$ formic acid. The flow rate was set to 400 $\mu \mathrm{L} /$ min. A gradient of mobile phase B was applied to ensure optimal separation of the analyzed lipid species. The mass spectrometer was operated in ESI-positive and -negative mode, capillary voltage $3500 \mathrm{~V}$ (positive) and $3000 \mathrm{~V}$ (negative), vaporize temperature $320^{\circ} \mathrm{C}$, ion transfer tube temperature $285 \mathrm{oC}$, sheath gas 60 arbitrary units, aux gas 20 arbitrary units and sweep gas 1 arbitrary unit. The Orbitrap MS scan mode at 120000 mass resolution was employed for lipid detection. The scan range was set to $250-1200 \mathrm{~m} / \mathrm{z}$ for both positive and negative ionization mode. The AGC target was set to $2.0 \mathrm{e} 5$ and the intensity threshold to 5.0e3. Data analysis was performed using the TraceFinder software (ThermoFisher Scientific). Lipidomics results from five biological replicates per group were analyzed. The amount of each lipid was calculated as concentration per mg of tissue for all lipid species, measured in a single biological replicate. Values were next averaged over the five biological replicates for control and MckCre-Gpcpd1 muscle samples, log2 transformed, and compared between the groups using lipidR software.

\section{Glycerophosphocholine and choline targeted metabolomics}

Choline and choline glycerophosphate were quantified by reversed phase chromatography on-line coupled to mass spectrometry, injecting $1 \mu \mathrm{l}$ of the methanolic serum extracts onto

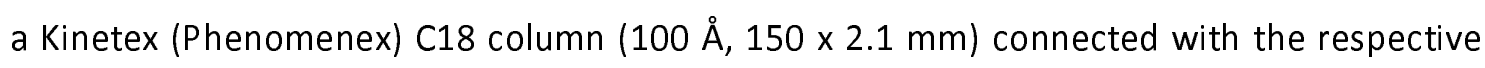


guard column. Metabolites were separated by employing a 7-minute-long linear gradient from $96 \%$ A (1 \% acetonitrile, $0.1 \%$ formic acid in water) to $80 \%$ B $(0.1 \%$ formic acid in acetonitrile) at a flow rate of $80 \mu \mathrm{l} / \mathrm{min}$. On-line tandem mass spectrometry (LC-MS/MS) was performed using the selected reaction monitoring (SRM) mode of a TSQ Altis mass spectrometer (Thermo Fisher Scientific), with the transitions $\mathrm{m} / \mathrm{z} 104.1 \rightarrow \mathrm{m} / \mathrm{z}$ 60.1, CE=20 (choline) and $\mathrm{m} / \mathrm{z} 258.1 \rightarrow \mathrm{m} / \mathrm{z}$ 104.1, CE=20 in the positive ion mode. Additionally, several amino acids (serine, isoleucine, leucine, tryptophan and phenylalanine) were recorded by respective Selected Reaction Monitoring (SRM) as internal standards. Data interpretation was performed using TraceFinder (Thermo Fisher Scientific). Authentic metabolite standards were used for validating experimental retention times by standard addition.

\section{Osmolarity measurements}

Quadriceps muscles were isolated from 8-week-old mice, snap frozen in liquid nitrogen, ground to a powder and weighed. Tissue lysates were prepared using metabolomics lysis buffer (20\% water/40\% Acetonytril/40\% Methanol) to reduce the interference of proteins on the measurements. The final solution was mixed with deionized water (1:3 ratio) and measured using K-7400S Semi-Micro Osmometer (Knauer, DE). Obtained measurements were normalized to tissue weight.

\section{Statistical analysis}

All data are expressed as mean $+/$ - standard error of the mean (SEM). Statistical significance was tested by Student's two tailed, unpaired t-test or two-way ANOVA followed by Sidak's multiple comparison test. All figures and statistical analyses were generated using Prism 8 (GraphPad) or R. Details of the statistical tests used are stated in each figure legend. In all figures, statistical significance is represented as *P<0.05, **P<0.01, ***P<0.001, ****P $<0.0001$. 


\section{Results}

\section{Aging affects the metabolic Gpcpd1-GPC pathway in the muscles}

We first set out to investigate if Gpcpd1 mRNA expression and GPC metabolite levels indeed change as a result of muscle ageing. We isolated skeletal muscles from adult (6 months) and old (24 months) mice. Gpcpd1 mRNA levels were markedly downregulated, while there was a highly significant accumulation of GPC in the aged muscles (Figure 1A, B). Further, we mined the recently reported multi-tissue age-dependent gene expression analysis in rats for potential changes in Gpcpd1 expression levels associated with ageing (Shavlakadze et al., 2019). Indeed, we found Gpcpd1 to be among the topmost significantly reduced genes in muscles with advanced age, displaying a high level of inverse correlation between Gpcpd1 mRNA levels and age $\left(R^{2}=0.5953 ; P<0.0001\right)$ (Figure $\left.1 C\right)$. This reduction was only evident in the skeletal muscle, while other tissues tested did not show such age-related Gpcpd1 mRNA decline (Supplementary Figure 1). These data show that Gpcpd1 expression is downregulated in the aged muscle of rodents, with a significant increase in its catalytic substrate GPC.

\section{Muscle specific Gpcpd1 deficiency does not affect muscle development but causes hyperglycemia in aged mice}

To assess the physiological function of Gpcpd1 in vivo, we generated Gpcpd1 $1^{\text {floxfflox }}$ mice to specifically delete Gpcpd1 in defined metabolically important tissues that display a high Gpcpd1 expression (Figure 1D; Supplementary Figure 2A). Successful gene targeting was confirmed by Southern Blot analysis (Figure 1E). As we found that Gpcpd1 expression was strongly reduced in aged muscles, we first crossed Gpcpd1 $1^{\text {flox/flox }}$ and MckCre transgenic mice to generate muscle-specific Gpcpd1 deficient offspring, here termed MckCre-Gpcpd1 $1^{\text {flox/flox }}$ mice. The efficiency of the Gpcpd1 deletion in the muscle of MckCre-Gpcpd1 $1^{\text {flox/flox }}$ animals was evaluated by RT-PCR. Gpcpd1 mRNA levels were significantly reduced, with some 
residual expression most likely resulting from non-myogenic cells (Figure 1F). Contrary to a previous report suggesting that over-expression of a truncated version of Gpcpd1 results in muscle atrophy (Okazaki et al., 2010), MckCre-Gpcpd1 flox/flox mice exhibited apparently normal muscle development, muscle morphology, and muscle sizes at 3-week old, 6 month old (Supplementary Figure 2B-D) and 20 months of age (Figure 1G,H). Intriguingly, aged MckCre-Gpcpd1 flox/flox mice (20 months old) developed fed and fasting hyperglycemia (Figure 1I). At the same time, there was a significant reduction in muscle glycogen levels (Figure 1J). Blood insulin levels remained unchanged (Figure 1K). These results indicate that muscle specific loss of Gpcpd1 has no apparent effect on muscle development or muscle mass in aging, but results in a muscle-mediated perturbation in glucose metabolism.

\section{Muscle-specific Gpcpd1 mutant mice display glucose intolerance.}

To directly address muscle glycemic control, we performed a glucose tolerance test in young (12-week-old) control and MckCre-Gpcpd1 $1^{\text {flox/flox }}$ mice. In contrast to older mice (Figure 1I) young MckCre-Gpcpd1 $1^{\text {flox/flox }}$ mice did not yet display fasting hyperglycemia (Supplementary Figure 2E). However, 12-week-old mutant mice already displayed a severe glucose intolerance (Figure 2A,B). At the same time, insulin release was unaffected (Figure 2C). Interestingly, this systemic metabolic dysfunction was apparent only due to muscle Gpcpd1 deficiency. 12-week-old liver-specific AlbCre-Gpcpd1 flox/flox (Figure 2D,E), white and brown fat-specific Ap2Cre-Gpcpd1 $1^{\text {floxfflox }}$ (Figure 2F,G), and brown fat-specific Upc1Cre-Gpcpd1 $1^{\text {floxfflox }}$ mice (Figure $2 \mathrm{H}, \mathrm{I}$ ) did not exhibit any apparent effects either on weight gain nor glucose metabolism, as addressed by glucose tolerance tests. Thus, muscle-specific Gpcpd1 deficiency results in glucose intolerance.

\section{Muscle-specific Gpcpd1 deficiency exacerbates high sugar and high fat diet induced metabolic syndrome}

To further examine the metabolic dysfunction that develops in MckCre-Gpcpd1 $1^{\text {floxfflox }}$ mice, the mice were first fed a high sugar diet (30\% fructose in drinking water). There were no detectable differences in weight gain between controls and MckCre-Gpcpd1 $1^{\text {flox flox }}$ mice over the 10 weeks observation period (Figure 3A). However, MckCre-Gpcpd $1^{\text {flox/flox }}$ mice displayed a severe defect in glucose clearance, as evaluated by a glucose tolerance test (Figure 3B). We next fed mice a high fat diet; again we observed no effects on weight gain but 
aggravated glucose intolerance of the MckCre-Gpcpd1 $1^{\text {flox/flox }}$ mice as compared to their control littermates (Figure 3C,D). Insulin release appeared unaffected under both high sugar and high fat diet conditions (Figure 3E,F). These data show that inactivation of Gpcpd1 in muscle does not affect weight gain under high sugar and fat diets, but markedly exacerbates glucose intolerance.

\section{Muscle-specific Gpcpd1 deficiency results in accumulation of glycerophosphocholine}

Since Gpcpd1 is responsible for hydrolysis of glyceroposphocholine (GPC), we reasoned that muscle Gpcpd1 deficiency would result in accumulation of GPC. To address this, we performed targeted lipidomic analysis on quadriceps muscle isolated from control and MckCre-Gpcpd1 flox/flox mice. As expected, there was a significant accumulation of GPC in the muscle of MckCre-Gpcpd1 $1^{\text {flox/flox }}$ mice (Figure 4A). Choline levels remained unchanged (Supplementary Figure 3A), which could be explain because choline can be provided in the food (Fagone and Jackowski, 2013). As GPC acts as an osmolyte (Gallazzini et al., 2008), we addressed whether the aberrant accumulation of GPC in muscles from MckCre-Gpcpd1 $1^{\text {flox/flox }}$ mice changes tissue osmolarity. Indeed, the osmolarity was significantly increased in muscles from MckCre-Gpcpd1 $1^{\text {floxfflox }}$ mice compared to control mice (Figure 4B). Similar changes were observed in muscles isolated from aged ( 24 months old) mice when compared to adult (6 months old) wild type mice (Figure $4 C$ ). Besides the marked increase in GPC, there were no significant changes in the global lipidome in the muscles from MckCreGpcpd1 $1^{\text {floxfflox }}$ mice (Supplementary Figure 3B), indicating that loss of Gpcpd1 specifically impairs degradation of GPC. Taken together, inactivation of Gpcpd1 in the muscle results in marked and specific accumulation of the lipid GPC and a change in tissue osmolarity.

\section{Muscle-specific Gpcpd1 deficiency impairs insulin signaling}

To obtain an overview of transcriptional changes occurring in muscles upon Gpcpd1 deficiency, we performed genome wide mRNA-sequencing analysis (Quant-Seq) to compare the skeletal muscle gene expression profiles from young (12 weeks old) MckCre-Gpcpd1 and control littermate mice (Figure 4C). Overall, 894 genes were significantly changed in skeletal muscles of MckCre-Gpcpd1 $1^{\text {flox/flox }}$ mice (401 down-regulated, 493 up-regulated; adjusted pvalue < 0.01). Interestingly, a large set of significantly dysregulated genes (Figure 4D and Supplementary Figure 4) were also the top-most dysregulated genes previously associated 
with muscle ageing (Shavlakadze et al., 2019). This data indicates that, at least partially, young MckCre-Gpcpd1 flox/flox mice display an "aged like" muscle transcriptional profile. Functional enrichment analysis of down-regulated genes in muscles of MckCre-Gpcpd1 $1^{\text {floxfflox }}$ mice revealed an enrichment for pathways linked to glucose and carbohydrate metabolism, and insulin signaling (adjusted p-value < 0.01) (Figure 4E). Indeed, MckCre-Gpcpd1 flox/flox mice displayed reduced insulin sensitivity as determined by an insulin tolerance test (ITT) (Figure 4F). Moreover, upon glucose challenge in vivo, we observed decreased phosphorylation of the insulin receptor beta chain (IRß) (Figure 4G). Thus, inactivation of Gpcpd1 in the muscle results in altered gene expression profiles associated with metabolic syndrome and aging and impaired Insulin receptor signaling.

\section{Elevated levels of GPCPD1 and GPC in aging and type 2 diabetes in humans}

We finally investigated how our findings relate to human physiology and aging (Krumpolec et al., 2020). We found that in the skeletal muscles of otherwise healthy, middle aged/old (49-62 years of age) individuals, there was a significant reduction in Gpcpd1 mRNA expression (Figure 5A). In parallel, there was a highly significant increase of GPC levels in skeletal muscles of sedentary aged individuals (Figure 5B,C), with levels of muscle GPC exponentially increasing with advanced age (Figure 5D). As we observed that deficiency of Gpcpd1 in mice results in impaired glucose metabolism, we also addressed if hyperglycemia in type 2 diabetic patients is associated with perturbed GPC metabolism. Indeed, there was a significant accumulation of GPC in skeletal muscles of diabetic individuals compared to age, gender and BMI matched controls (Figure 5E), while several other metabolites remained unchanged (Figure 5F). Taken together, our data show that the GPCPD1-GPC metabolic axis is dysregulated with aging in humans, and that GPC accumulates in muscles of type 2 diabetes patients.

\section{Discussion}

Muscle aging is accompanied by a myriad of molecular and metabolic changes. Yet, it still remains elusive which of these perturbations are causative to the aging process, affecting both the muscle and systemic health (Demontis et al., 2013b). Here, we report that the 
Gpcpd1-GPC metabolic axis is severely perturbed with aging in muscles of rodents and humans. Based on these observations, we generated conditional and subsequently tissuespecific Gpcpd1 deficient mice to investigate if age-related changes in this metabolic pathway affect systemic health. We found that muscle specific Gpcpd1 deficiency results in a severe glucose intolerance, a disorder that is commonly seen in the elderly (Chia et al., 2018). Thus, age-related perturbations of the Gpcpd1-GPC metabolic pathway are critically involved in altered glucose metabolism in aging.

Gpcpd1 deficiency has no apparent effect on body weight, muscle development, nor muscle mass even in old age. The apparently normal muscle mass in our model contrasts with a previous report where overexpression of a truncated Gpcpd1 protein in vivo resulted in muscle atrophy (Okazaki et al., 2010). This could be attributed to high levels of transgene expression, which can result in cell and tissue toxicity (Bolognesi and Lehner, 2018; Kulak et al., 2014; Moriya, 2015), not excluding other reasons for this previously observed phenotype such as dominant-negative effects of the overexpressed protein in defined pathways. We also deleted Gpcpd1 specifically in several other metabolically important mouse tissues with a Gpcpd1 expression; however, we never observed any gross-developmental defects nor impairment in glucose metabolism. Although we cannot rule out roles of Gpcpd1-GPC in these tissues or in tissues we have not assessed yet, our results show that muscle-regulated glucose homeostasis is critically dependent on Gpcpd1 expression.

Mechanistically, our data show that Gpcpd1 deficiency inhibits muscle glucose uptake by interfering with glucose metabolism and insulin signaling. How would high levels of GPC, the specifically increased substrate of Gpcpd1, affect these pathways? It has been shown that the excess accumulation of other lipid species in the skeletal muscle, such as diacylglycerol, triacylglycerol, and ceramides, impairs insulin sensitivity and glucose uptake by perturbing the mitochondrial beta oxidation and triggering the inflammatory program (Park and Seo, 2020; Wu and Ballantyne, 2017). It's therefore possible that GPC exerts similar effects, although we didn't find any signs of muscle inflammation in MckCre-Gpcpd1 $1^{\text {floxfflox }}$ mice. It has been previously reported that prolonged exposure to osmotic shock dramatically decreases insulin stimulated glucose uptake and glycogen synthesis (Chen et al., 1999; Gual et al., 2003; Stookey et al., 2004). On the other hand, renal medullary epithelial cells rapidly 
increase and decrease GPC levels to counteract osmotic changes (Gallazzini et al., 2008). Increased myofiber osmolarity has been observed in muscles of the elderly (Lim et al., 2019; Ritz et al., 2000), which we confirmed and also detected in muscle tissue of Gpcpd1 mutant mice and in older wild type animals. In parallel, both aged muscles and Gpcpd1 deficient muscles in younger mice displayed persistently increased levels of GPC, which we also observed in older humans and people diagnosed with type 2 diabetes. Whether these changes in myofiber osmolarity interfere with insulin signaling or glucose transport into the muscle cells, thus impairing glucose clearance by the muscle and resulting in systemic defects of glucose metabolism, needs to be determined. It remains to be elucidated why and how aging and diabetes result in reduced Gpcpd1 in muscles, and how such perturbation of the Gpcpd1-GPC metabolic pathway can be reverted to preserve the muscle-mediated regulation of metabolic glucose homeostasis in the elderly.

Conclusions. Our data provide evidence that ageing results in a perturbed Gpcpd1-GPC metabolic axis in the muscle. Muscle specific Gpcpd1 deficient mice display systemic glucose intolerance, without apparently affecting muscle development and size. Assessing tissue specific deletion of Gpcpd1 in several organs that are of key importance to whole-body metabolic glucose control, we further show that this effect on glucose intolerance and hyperglycemia appears to be muscle specific. Mechanistically, we find that specific accumulation of GPC due to loss of Gpcpd1 in the muscle perturbs muscle carbohydrate metabolism, myofiber osmolarity, and insulin signaling and is associated with an "aged like" transcriptional profile. Finally, we report that in the muscles of aged humans as well as of type 2 diabetes patients, the GPCPD1-GPC axis is markedly changed. Thus, in mice and men we have identified a critical pathway in muscle that regulates systemic glucose homeostasis in aging. 


\section{Author contributions}

D.C. and J.M.P. conceived, coordinated, and designed the study. D.C. and M.O. performed experiments and analyzed the data with contributions from M.L. and S.J.F. M.N. performed bioinformatic analysis. A.H. assisted in tissue sampling. E.R. and T.G. collected human muscle biopsies. L.P., R.K. and M.Krs. performed and analyzed in vivo MRS measurements. M.L., M.K., M.Krs. and A.K.-W. designed, coordinated and oversaw the human T2DM experiments. D.C. and J.M.P. wrote the manuscript. All authors edited the manuscript and approved the final manuscript.

\section{Acknowledgements}

We would like to thank all members of our laboratories for helpful discussions. We are grateful to Vienna Biocenter Core Facilities: Transgenic unit, Comparative medicine and Metabolomics unit for their service. We also than the lipidomic service of Center for Molecular Medicine (CEMM). We would like to thank Dr. Patrik Krumpolec from the Biomedical Research Center, Institute of Experimental Endocrinology, Slovak Academy of Sciences, Bratislava, Slovakia, for the processing of MRS data from young and senior participants. We would like to thank Prof. S. Trattnig, High Field MR Centre, Department of Biomedical Imaging and Image guided Therapy for logistic support. J.M.P. is supported by IMBA, a Wittgenstein award, the T. von Zastrow foundation, and a Canada 150 Research Chair in functional genetics. D.C. is supported by the Austrian Academy of Sciences and the T. von Zastrow foundation. M. Krs., R. K. and L. P. are supported by Austrian Science Foundation (KLI-904-B).

\section{Conflict of interest}

The authors have declared that no conflict of interest exists. 


\section{References}

Baskin, K.K., Winders, B.R., and Olson, E.N. (2015). Muscle as a "mediator" of systemic metabolism. Cell Metab. 21.

Beiglböck, H., Wolf, P., Pfleger, L., Caliskan, B., Fellinger, P., Zettinig, G., Anderwald, C.H., Kenner, L., Trattnig, S., Kautzky-Willer, A., et al. (2020). Effects of thyroid function on phosphodiester concentrations in skeletal muscle and liver: An in vivo nmrs study. J. Clin. Endocrinol. Metab. 105.

Bergström, J., and Hultman, E. (1967). A study of the glycogen metabolism during exercise in man. Scand. J. Clin. Lab. Invest.

Bolognesi, B., and Lehner, B. (2018). Protein Overexpression: Reaching the limit. Elife 7.

Chen, D., Fucini, R. V., Olson, A.L., Hemmings, B.A., and Pessin, J.E. (1999). Osmotic Shock Inhibits Insulin Signaling by Maintaining Akt/Protein Kinase B in an Inactive Dephosphorylated State. Mol. Cell. Biol. 19.

Chia, C.W., Egan, J.M., and Ferrucci, L. (2018). Age-related changes in glucose metabolism, hyperglycemia, and cardiovascular risk. Circ. Res. 123.

Corda, D., Mosca, M.G., Ohshima, N., Grauso, L., Yanaka, N., and Mariggiò, S. (2014). The emerging physiological roles of the glycerophosphodiesterase family. FEBS J. 281.

Cowie, C.C., Rust, K.F., Ford, E.S., Eberhardt, M.S., Byrd-Holt, D.D., Li, C., Williams, D.E., Gregg, E.W., Bainbridge, K.E., Saydah, S.H., et al. (2009). Full accounting of diabetes and pre-diabetes in the U.S. population in 1988-1994 and 2005-2006. Diabetes Care 32.

Cruz-Jentoft, A.J., and Sayer, A.A. (2019). Sarcopenia. Lancet 393.

DeFronzo, R.A., Jacot, E., Jequier, E., Maeder, E., Wahren, J., and Felber, J.P. (1981). The effect of insulin on the disposal of intravenous glucose. Results from indirect calorimetry and hepatic and femoral venous catheterization. Diabetes 30 .

Demontis, F., Piccirillo, R., Goldberg, A.L., and Perrimon, N. (2013a). Mechanisms of skeletal muscle aging: Insights from Drosophila and mammalian models. DMM Dis. Model. Mech. 6.

Demontis, F., Piccirillo, R., Goldberg, A.L., and Perrimon, N. (2013b). The influence of skeletal muscle on systemic aging and lifespan. Aging Cell 12.

Fagone, P., and Jackowski, S. (2013). Phosphatidylcholine and the CDP-choline cycle. Biochim. Biophys. Acta - Mol. Cell Biol. Lipids 1831.

Gallazzini, M., Ferraris, J.D., and Burg, M.B. (2008). GDPD5 is a glycerophosphocholine phosphodiesterase that osmotically regulates the osmoprotective organic osmolyte GPC. Proc. Natl. Acad. Sci. U. S. A. 105.

Gual, P., Gonzalez, T., Grémeaux, T., Barrés, R., Le Marchand-Brustel, Y., and Tanti, J.F. (2003). Hyperosmotic stress inhibits insulin receptor substrate-1 function by distinct mechanisms in 3T3-L1 adipocytes. J. Biol. Chem. 278.

Houtkooper, R.H., Argmann, C., Houten, S.M., Canto, C., Jeninga, E.H., Andreux, ?eńelope A., Thomas, C., Doenlen, R., Schoonjans, K., and Auwerx, J. (2011). The metabolic footprint of aging in mice. Sci. Rep. 1.

Jue, T., Rothman, D.L., Shulman, G.I., Tavitian, B.A., DeFronzo, R.A., and Shulman, R.G. (1989). Direct observation of glycogen synthesis in human muscle with 13C NMR. Proc. Natl. Acad. Sci. U. S. A. 86. 
Kern, M., Dolan, P.L., Mazzeo, R.S., Wells, J.A., and Dohm, G.L. (1992). Effect of aging and exercise on GLUT-4 glucose transporters in muscle. Am. J. Physiol. - Endocrinol. Metab. 263.

Krumpolec, P., Klepochová, R., Just, I., Tušek Jelenc, M., Frollo, I., Ukropec, J., Ukropcová, B., Trattnig, S., Krššák, M., and Valkovič, L. (2020). Multinuclear MRS at 7T Uncovers Exercise Driven Differences in Skeletal Muscle Energy Metabolism Between Young and Seniors. Front. Physiol. 11.

Kulak, N.A., Pichler, G., Paron, I., Nagaraj, N., and Mann, M. (2014). Minimal, encapsulated proteomic-sample processing applied to copy-number estimation in eukaryotic cells. Nat. Methods 11.

Lattin, J.E., Schroder, K., Su, A.I., Walker, J.R., Zhang, J., Wiltshire, T., Saijo, K., Glass, C.K., Hume, D.A., Kellie, S., et al. (2008). Expression analysis of G Protein-Coupled Receptors in mouse macrophages. Immunome Res. 4.

Lim, J.Y., Choi, S.J., Widrick, J.J., Phillips, E.M., and Frontera, W.R. (2019). Passive force and viscoelastic properties of single fibers in human aging muscles. Eur. J. Appl. Physiol. 119.

Moriya, H. (2015). Quantitative nature of overexpression experiments. Mol. Biol. Cell 26.

Okazaki, Y., Ohshima, N., Yoshizawa, I., Kamei, Y., Mariggiò, S., Okamoto, K., Maeda, M., Nogusa, Y., Fujioka, Y., Izumi, T., et al. (2010). A novel glycerophosphodiester phosphodiesterase, GDE5, controls skeletal muscle development via a non-enzymatic mechanism. J. Biol. Chem. 285.

Park, S.S., and Seo, Y.K. (2020). Excess accumulation of lipid impairs insulin sensitivity in skeletal muscle. Int. J. Mol. Sci. 21.

Pilling, L.C., Atkins, J.L., Bowman, K., Jones, S.E., Tyrrell, J., Beaumont, R.N., Ruth, K.S., Tuke, M.A., Yaghootkar, H., Wood, A.R., et al. (2016). Human longevity is influenced by many genetic variants: Evidence from 75,000 UK Biobank participants. Aging (Albany. NY). 8.

Ritz, P., Fellmann, N., Levadoux, E., Morio, B., Vaché, C., Ritz, P., Arnaud, M.J., Acher, S., Beaufrère, B., Blonde-Cynober, F., et al. (2000). Body water spaces and cellular hydration during healthy aging. In Annals of the New York Academy of Sciences, $p$.

Shavlakadze, T., Morris, M., Fang, J., Wang, S.X., Zhu, J., Zhou, W., Tse, H.W., Mondragon-Gonzalez, R., Roma, G., and Glass, D.J. (2019). Age-Related Gene Expression Signature in Rats Demonstrate Early, Late, and Linear Transcriptional Changes from Multiple Tissues. Cell Rep.

Stewart, J.D., Marchan, R., Lesjak, M.S., Lambert, J., Hergenroeder, R., Ellis, J.K., Lau, C.H., Keun, H.C., Schmitz, G., Schiller, J., et al. (2012). Choline-releasing glycerophosphodiesterase EDI3 drives tumor cell migration and metastasis. Proc. Natl. Acad. Sci. U. S. A. 109.

Stookey, J., Pieper, C., and Cohen, H. (2004). Hypertonic hyperglycemia progresses to diabetes faster than normotonic. Eur. J. Epidemiol. 19.

Valkovič, L., Ukropcová, B., Chmelík, M., Baláž, M., Bogner, W., Schmid, A.I., Frollo, I., Zemková, E., Klimeš, I., Ukropec, J., et al. (2013). Interrelation of 31P-MRS metabolism measurements in resting and exercised quadriceps muscle of overweight-to-obese sedentary individuals. NMR Biomed. 26.

Wu, H., and Ballantyne, C.M. (2017). Skeletal muscle inflammation and insulin resistance in obesity. J. Clin. Invest. 127.

Wu, C., Jin, X., Tsueng, G., Afrasiabi, C., and Su, A.I. (2016). BioGPS: Building your own mash-up of gene annotations and expression profiles. Nucleic Acids Res. 44.

Yanagi, N., Koike, T., Kamiya, K., Hamazaki, N., Nozaki, K., Ichikawa, T., Matsunaga, A., Kuroiwa, M., and Arai, M. (2021). Assessment of sarcopenia in the intensive care unit and 1-year mortality in 
bioRxiv preprint doi: https://doi.org/10.1101/2021.10.26.465828; this version posted October 26, 2021. The copyright holder for this preprint (which was not certified by peer review) is the author/funder. All rights reserved. No reuse allowed without permission.

survivors of critical illness. Nutrients 13.

Yang, M., Jiang, J., Zeng, Y., and Tang, H. (2019). Sarcopenia for predicting mortality among elderly nursing home residents: SARC-F versus SARC-CalF. Med. (United States) 98. 


\section{Figure legends}

\section{Figure 1. Muscle-specific Gpcpd1 deficiency causes hyperglycemia in old mice.}

A) mRNA levels in quadriceps muscles of adult ( 6 months old) and old (24 months old) mice. $\mathrm{N}=5$ per group. B) Glycerophosphocholine (GPC) levels in quadriceps muscles of young/adult (6 months old) and old (24 months old) mice. $\mathrm{N}=5-10$ per group. C) Correlation of mRNA expression of Gpcpd1 in rat muscles with age. D) Schematic outline and E) Southern blot validation of successful generation of a conditional Gpcpd1 allele in mice. F) Validation of Gpcpd1 mRNA deletion in muscles from muscle-specific Gpcpd1 mutant mice (MckCreGpcpd1 $1^{\text {flox/flox }}$ ). N=6 per group. G) Bodyweight of 20 months old control and littermate MckCre-Gpcpd1 flox/flox mice. N=7-8 per group. $\mathrm{H}$ ) Weights of skeletal muscle (quadriceps, $\mathrm{QA}$; gastrocnemicus, GC; and Tibialis anterior, TA) isolated from 20 months old control and MckCreGpcpd flox/flox mice. $\mathrm{N}=7$ per group. I) Blood glucose levels of 20 months old littermate control and MckCre-Gpcpd1 $1^{\text {flox/flox }}$ mice under fasted and re-fed states ( $2 \mathrm{~h}$ after re-feeding; standard diet). N=6-7 per group. J) Skeletal muscle (quadriceps) glycogen levels in 20 months old control and Mck-CreGpcpd $1^{\text {flox/flox }}$ mice. $\mathrm{N}=6$. K) Blood insulin levels of standard diet fed 20 months old control and MckCre-Gpcpd1 $1^{\text {flox/flox }}$ mice. $\mathrm{N}=6-7$ per group. Unless otherwise stated, each dot represents an individual mouse. Data are shown as means \pm SEM. Student's two tailed, unpaired t-test was used for statistical analysis; ns, not significant; ${ }^{*} p<0.05 ; * * p$ $<0.01 ; * * * p<0.001, * * * * p<0.0001$. please adjust where ns stands in the figures and maybe also add a bar like for the **

Figure 2. Muscle Gpcpd1 deficiency causes glucose intolerance in young mice.

A) Blood glucose levels and B) Area under curve (AUC) after an oral glucose tolerance test (OGTT) in 2-3 months old control and MckCre-Gpcpd1 $1^{\text {floxfflox }}$ mice. $\mathrm{N}=16-17$ per group. Student's two tailed un-paired t test was used for AUC statistical analysis. C) Blood insulin levels during OGTT in 2-3 months old control and MckCre-Gpcpd1 $1^{\text {flox/flox }}$ mice. $\mathrm{N}=12$ per group. D) Body weights of 3 months old control and hepatocyte-specific Gpcpd1 deficient (AlbCre-Gpcpd1 $1^{\text {flox/flox }}$ ) littermates. N=17-20 per group. E) Blood glucose levels after OGTT 
test in 2-3 months old control and AlbCre-Gpcpd1 flox/flox mice. $\mathrm{N}=17-20$ per group. F) Body weights of 3 months old control and white and brown fat-specific Gpcpd1 deficient (Ap2CreGpcpd1 $1^{\text {flox/flox }}$ ) littermates. N=4-6 per group. G) Blood glucose levels after OGTT in 2-3 months old control and Ap2Cre-Gpcpd1 $1^{\text {floxfflox }}$ mice. N=4-6 per group. H) Body weights of 3 months old control and brown fat-specific Gpcpd1 deficient (Ucp1Cre-Gpcpd1 flox/flox) littermates. N=4-6 per group. I) Blood glucose levels after OGTT test in 2-3 months old control and Ucp1Cre-Gpcpd $1^{\text {flox fflox }}$ mice. N=4-6 per group. Unless otherwise stated, each dot represents an individual mouse. Data are shown as means \pm SEM. Two-way ANOVA followed by Sidak's multiple comparison test was used for statistical analysis unless otherwise stated; ns, not significant; ${ }^{*} p<0.05 ; * * p<0.01 ; * * * p<0.0001$.

\section{Figure 3. Muscle specific Gpcpd1 deficiency exacerbates high sugar and high fat diet induced metabolic syndrome.}

A) Body weight gain of 3 months old control and MckCre-Gpcpd flox/flox mice on high carbohydrate diet (HCD; 30\% fructose). N=7 per group. B) Blood glucose levels after OGTT in 3 months old control and MckCre-Gpcpd1 $1^{\text {flox/flox }}$ mice fed a HCD diet. N=7 per group. C) Body weight gain of 3 months old control and MckCre-Gpcpd1 $1^{\text {floxfflox }}$ mice on high fat diet (HFD; $60 \%$ fat). $\mathrm{N}=9$ per group. D) Blood glucose levels after OGTT in 3 months old control and MckCre-Gpcpd1 flox/flox littermate mice fed a HFD diet. $\mathrm{N}=9$ per group. E) Blood insulin levels during OGTT in 3 months old control and MckCre-Gpcpd flox/flox mice fed HCD. N=7 per group. F) Blood insulin levels during OGTT in 3 months old control and MckCre-Gpcpd1 mice fed HFD. $\mathrm{N}=9$ per group. Unless otherwise stated, each dot represents an individual mouse. Data are shown as means \pm SEM. Two-way ANOVA followed by Sidak's multiple comparison test was used for statistical analysis unless otherwise stated; ns, not significant; *p $<0.05,{ }^{* *} p<$ 0.01 .

\section{Figure 4. Muscle aging gene expression signatures and impaired Insulin receptor signaling.}

A) Glycerophosphocholine (GPC) levels in skeletal muscle (quadriceps) of 3 months old control and MckCre-Gpcpd1 $1^{\text {floxfflox }}$ mice. $\mathrm{N}=10-12$ per group. B) Skeletal muscle tissue 
osmolarity (quadriceps) of 3 months old control and MckCre-Gpcpd1 $1^{\text {flox/flox }}$ mice. N=6-7 per group. C) Skeletal muscle tissue osmolarity (quadriceps) of 6 months old and 24 months old wild type mice. $\mathrm{N}=5-10$ per group. D) Quantseq transriptomic analysis in skeletal muscle (gastrocnemicus) of 3 months old control and MckCre-Gpcpd1 flox/flox littermate mice. $\mathrm{N}=4$ per group. E) Gene ontology analysis of down-regulated genes in skeletal muscle from 3 months old MckCre-Gpcpd1 $1^{\text {floxfflox }}$ mice, compared to control littermates. F) Insulin tolerance test of 3 months old control and MckCre-Gpcpd1 $1^{\text {floxfflox }}$ mice. $\mathrm{N}=16-17$ per group. Mice were fasted $6 \mathrm{~h}$ before the test. $G$ ) Insulin receptor beta (IR $\beta$ ) signaling in skeletal muscles (quadriceps) from two individual, 3 months old control and MckCre-Gpcpd1 $1^{\text {floxfflox }}$ mice after overnight fasting and 15 minutes after oral glucose delivery (bolus $1 \mathrm{~g}$ glucose $/ \mathrm{kg}$ of body weight). Total IR $\beta$ levels and phosphorylated IR (phosphoTyr1150/Tyr1151 IR $\beta$ are shown. GAPDH is shown as a loading control. Unless otherwise stated, each dot in A-C represents an individual mouse. Data are shown as means \pm SEM. Student's two tailed, unpaired t-test was used for statistical analysis; ns, not significant. ${ }^{*} \mathrm{p}<0.05$.

\section{Figure 5. The metabolic GPCPD1-GPC axis is perturbed with aging and type 2 diabetes in humans.}

A) Gpcpd1 mRNA levels in quadriceps muscles in otherwise healthy young (20-30yrs) and old (49-65) humans. Relative mRNA expression normalized to 18S RNA is shown. B) Representative ${ }^{31} \mathrm{P}$-magnetic resonance (MR) spectra acquired from the skeletal muscle (gastrocnemius medialis) from young (20-30yrs) and senior (49-65) participants. C) GPC levels in the skeletal muscle from young (20-30yrs) and old (49-65) participants measured by

${ }^{31} \mathrm{P}$ magnetic resonance spectroscopy (MRS). D) Levels of muscle GPC levels plotted against age for each individual. Linear-regression analysis was used for $\mathrm{R} 2$ and $\mathrm{P}$ values calculation. E) Representative ${ }^{31}$ P-MR spectra, GPC and GPC-PDE levels acquired from the skeletal muscle (gastrocnemius medialis) from healthy volunteers and type 2 diabetes patients (see Methods for cohort description). F) Levels of phosphocreatine ( $\mathrm{PCr}$ ), inorganic phosphate (Pi), phosphodiester (PDE), phosphocholine (PC), phosphomonoesthers (PME) acquired from the skeletal muscle from healthy volunteers and type 2 diabetes patients. Unless otherwise stated, each dot represents an individual human. Data are shown as means \pm SEM. Student's 
two tailed, unpaired t-test was used for statistical analysis unless otherwise stated; ns, not significant; $* \mathrm{p}<0.05, * * * \mathrm{p}<0.001, * * * \mathrm{p}<0.0001$.

\section{Supplementary Figure legends}

\section{Supplementary Figure 1. Assessment of age-related Gpcpd1 levels in rat tissues.}

Correlation of mRNA expression of Gpcpd1 in the indicated rat tissues with age. Data are shown as means \pm SEM. Student's two tailed, unpaired t-test was used for statistical analysis unless otherwise stated. There were no statistically significant differences.

\section{Supplementary Figure 2. Muscle weights assessment and fasting glucose levels in young Mck-CreGpcpd ${ }^{\text {flox/flox }}$ mice.}

A) Cross-tissue Gpcpd1 expression analysis in mice. B,C) Skeletal muscle weights of B) 3 weeks old (quadriceps) and C) 3 months old (quadriceps, QA; gastrocnemicus, GC; and Tibialis anterior, TA) control and $M c k-C r e G p c p d 1^{\text {flox/flox }}$ mice. $\mathrm{N}=6$ and $\mathrm{N}=7$ per group, respectively. D) Cross section of quadriceps muscle from 6 months old control and MckCreGpcpd1 $1^{\text {floxfflox }}$ mice stained for dystrophin (green) and nuclei (blue). No apparent structural defects were observed in the muscles of Mck-CreGpcpd1 $1^{\text {flox/flox }}$ mice. E) Fasting blood glucose levels in 3 months old control and Mck-CreGpcpd1 $1^{\text {flox/flox }}$ mice. $\mathrm{N}=6$ and $\mathrm{N}=7$ per group. Each dot represents one individual animal. Data are shown as means \pm SEM. Student's two tailed, unpaired t-test was used for statistical analysis unless otherwise stated, ns, not significant.

\section{Supplementary Figure 3. No significant changes in the muscle lipidome upon} Gpcpd1 deficiency.

A) Choline levels in skeletal muscle (quadriceps) of 3 months old control and $\mathrm{MckCre-}$ Gpcpd1 $1^{\text {flox/flox }}$ mice. $\mathrm{N}=10-12$ per group. Each dot represents one individual animal. B) Mass spectrometry based un-targeted lipidomic analysis in quadriceps muscles isolated from 3 
months old control and Mck-Cre-Gpcpd1 flox/flox littermate mice. Abbreviations denote triacylglycerols (TAG), sphingomyelins (SM), phosphatidylinositols (PI), phosphatydilethanolamines (PE), phosphatydilcholines (PC), lysophosphatydilethanolamines (LPE), lysophosphatydilcholines (LPC), diacylglycerols (DAG), ceramides (Cer), and cholesterolesther (CE). Numbers denote the carbon numbers, heatmap denotes higher and lower abundant lipid species in the muscles. No significant differences were found in the indicated lipid species abundance between muscles isolated from control and Mck-CreGpcpd1 $1^{\text {flox/flox }}$ mice. $\mathrm{N}=5$ per group. Data are shown as means \pm SEM. Student's two tailed, unpaired t-test was used for statistical analysis unless otherwise stated, ns, not significant.

\section{Supplementary Figure 4. Muscles from young Mck-CreGpcpd1 ${ }^{\text {flox/flox }}$ mice display an "aged-like" transcriptomic signature.}

Comparison of upregulated (red) and downregulated (blue) gene expression changes in aged skeletal muscles in rats (27 month old versus 6 month old) (Shavlakadze et al., 2019) with gene expression changes of skeletal muscles from 3 month old control and MckCreGpcpd $1^{\text {flox/flox }}$ mice. Overlapping dysregulated genes are highlighted. 


\section{Figure 1}
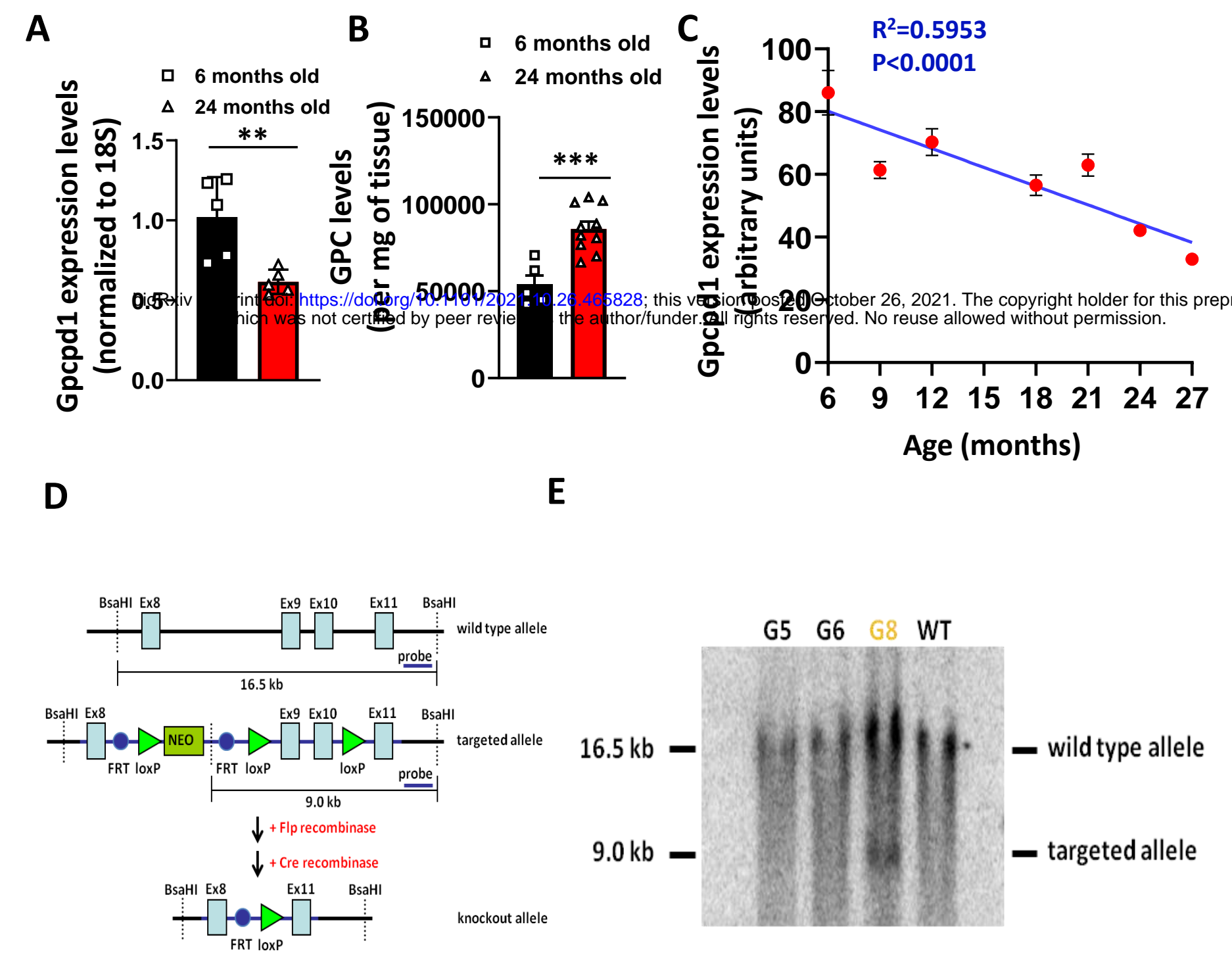

F

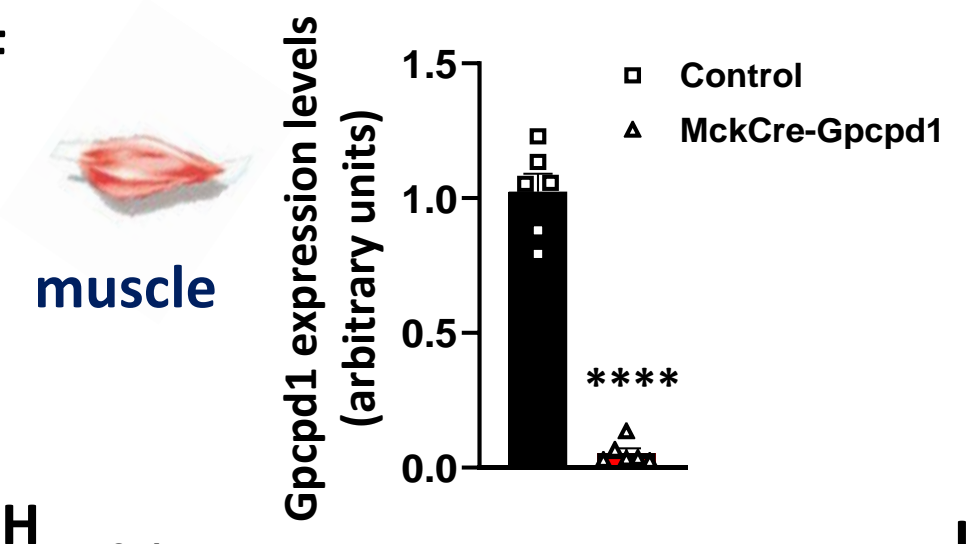

G
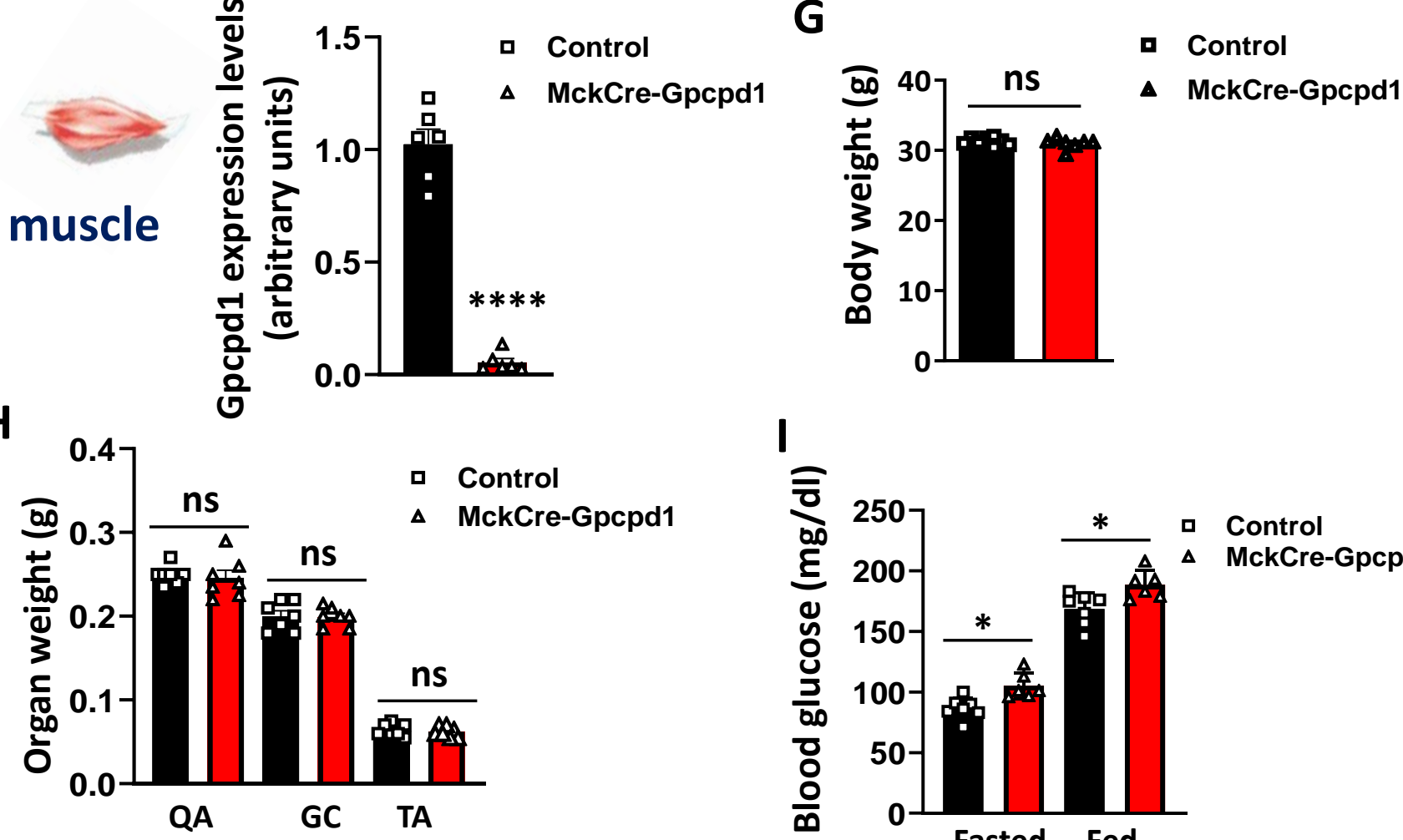

1
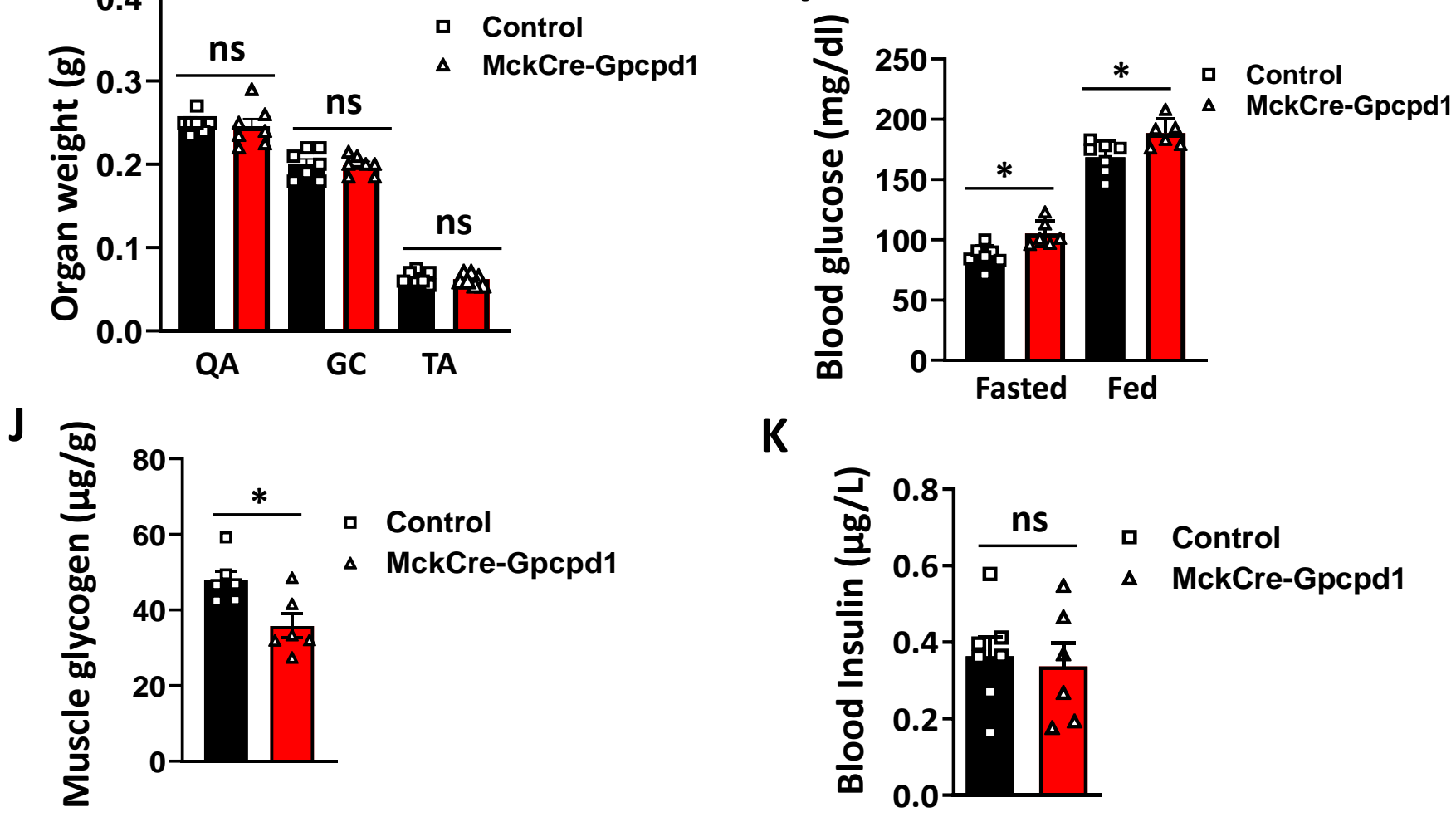

K

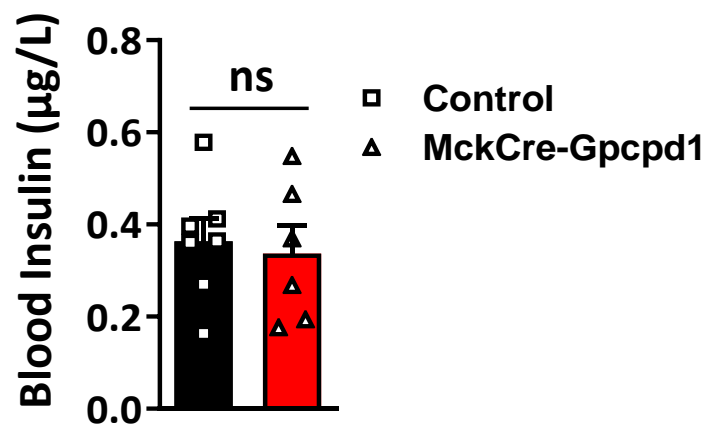


Figure 2

A

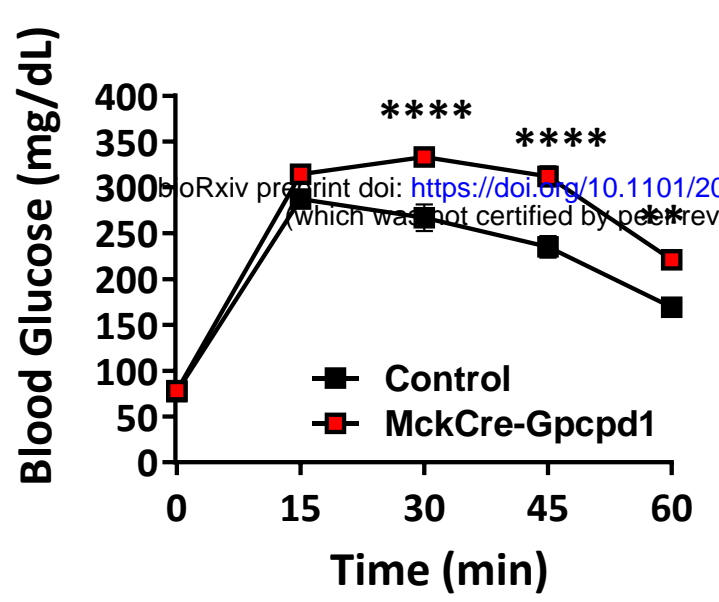

B

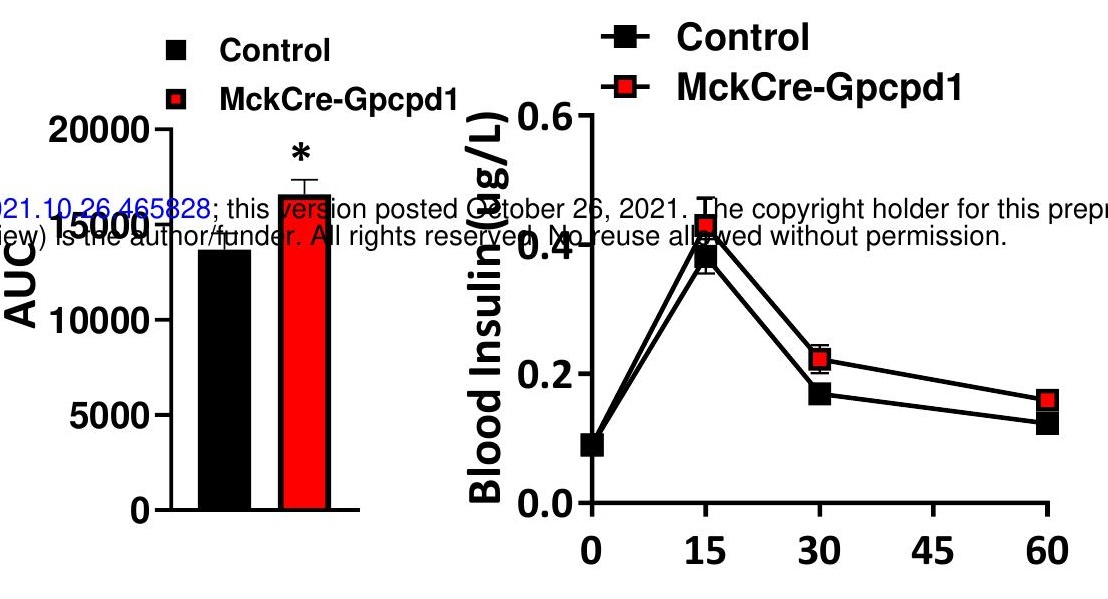

D

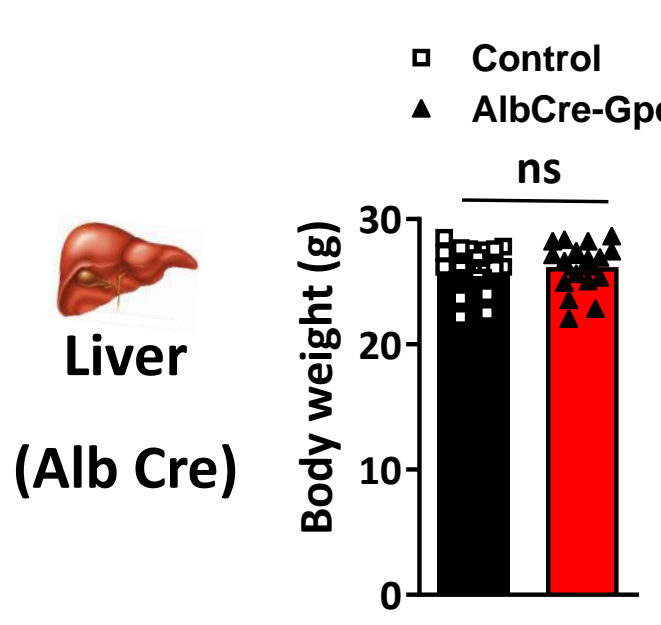

F

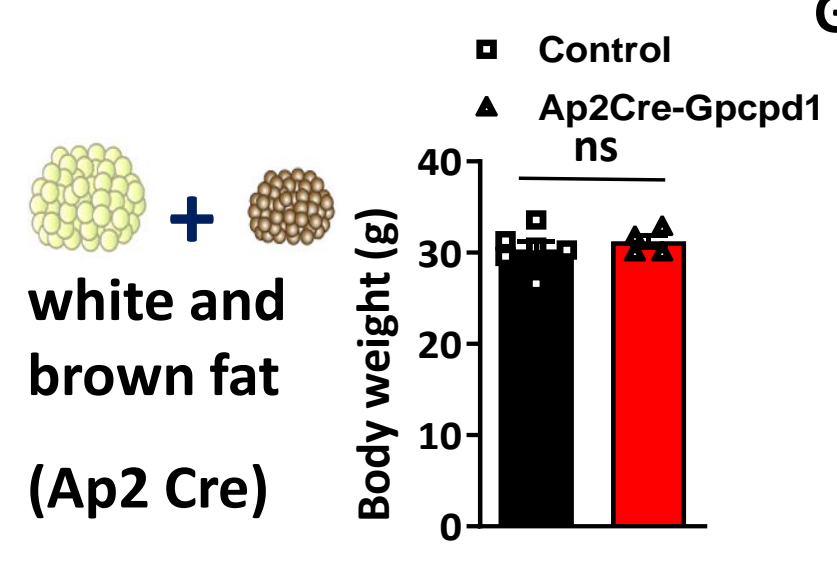

H

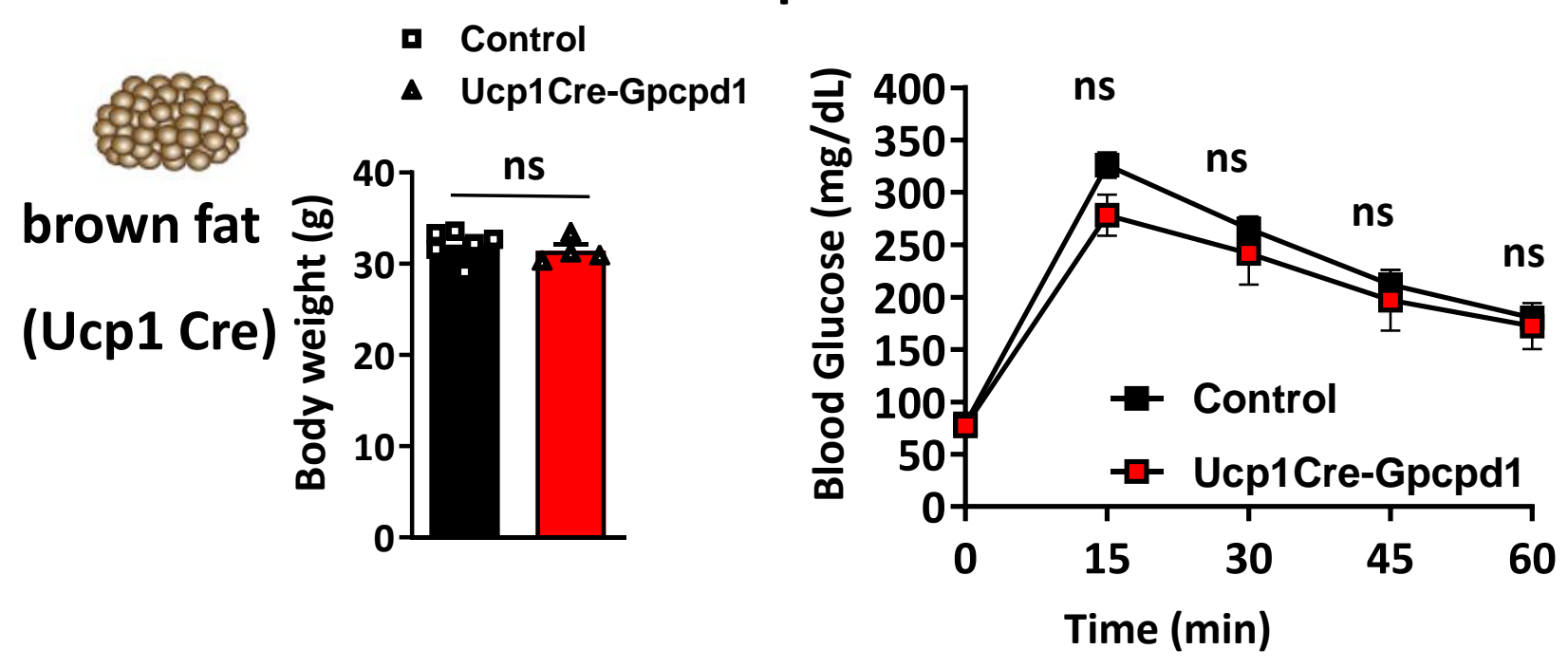

E

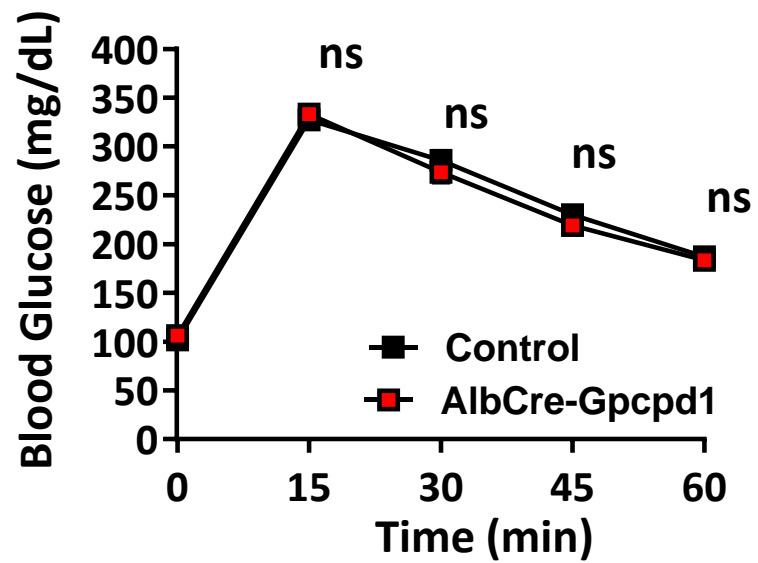

G

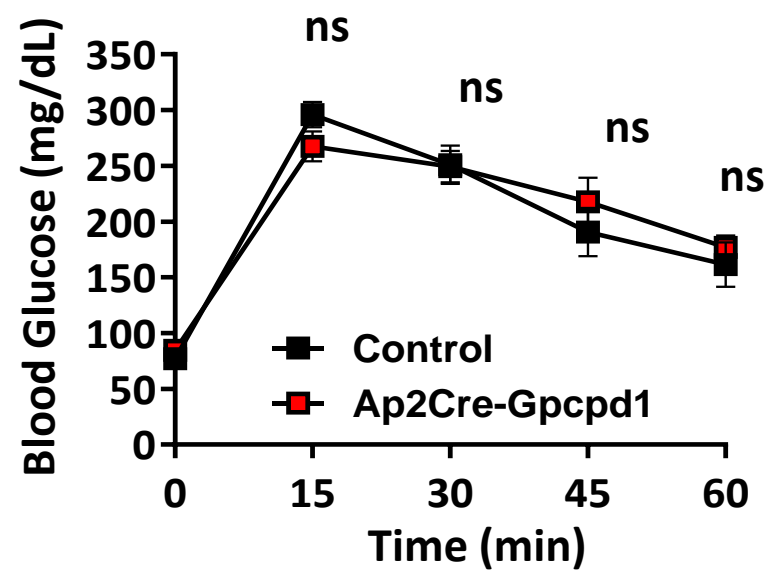

I 
Figure 3

A

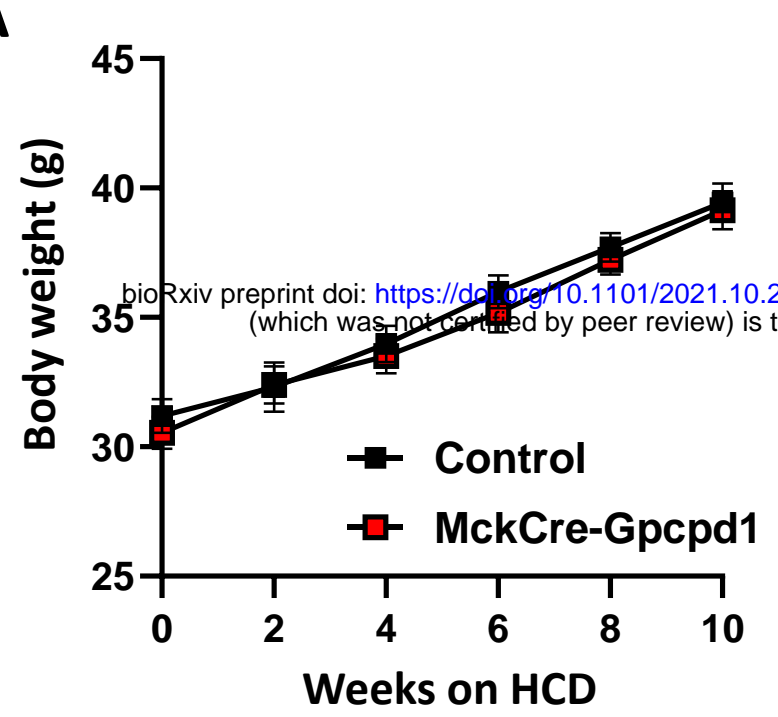

C

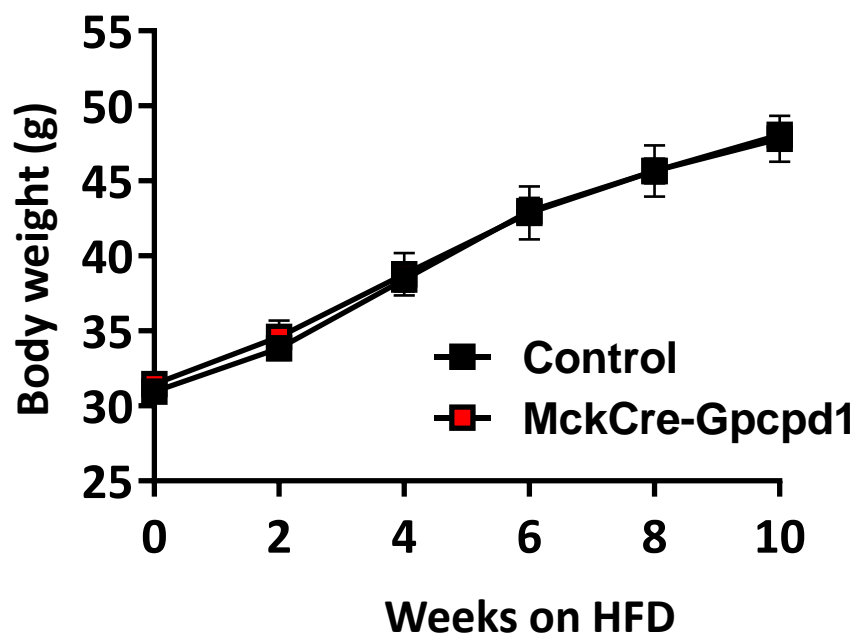

E

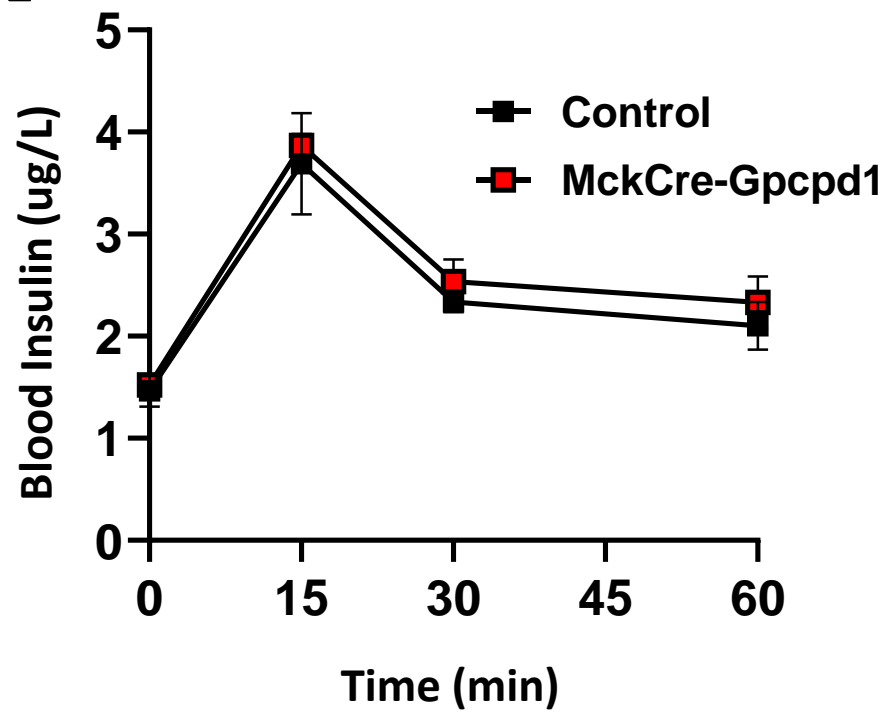

B
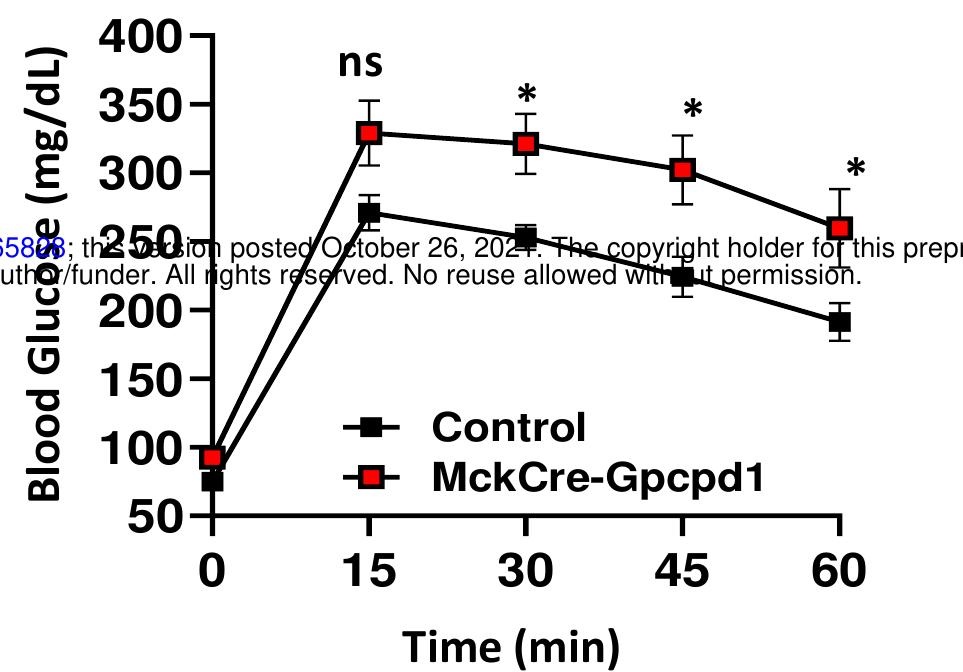

D

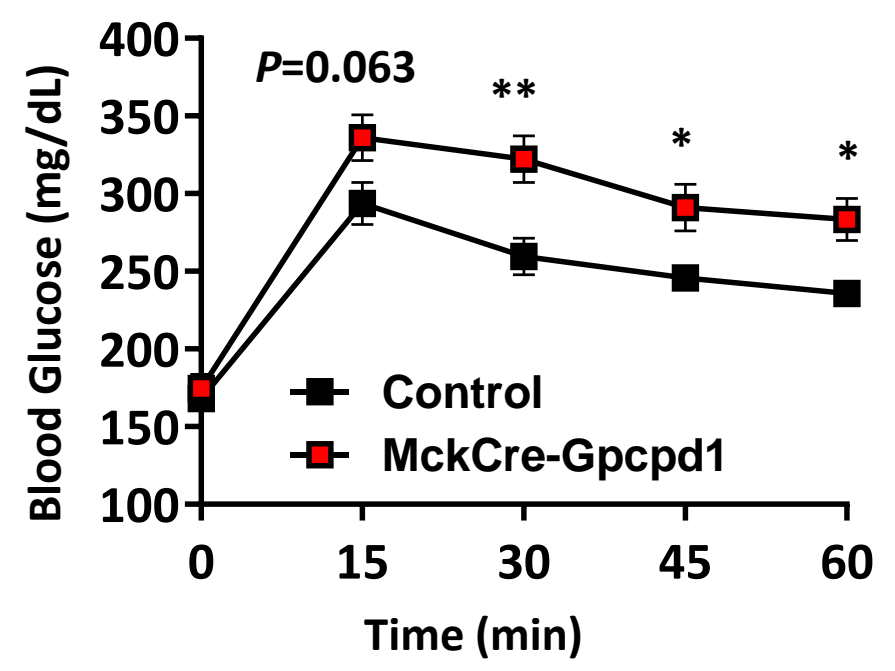

F

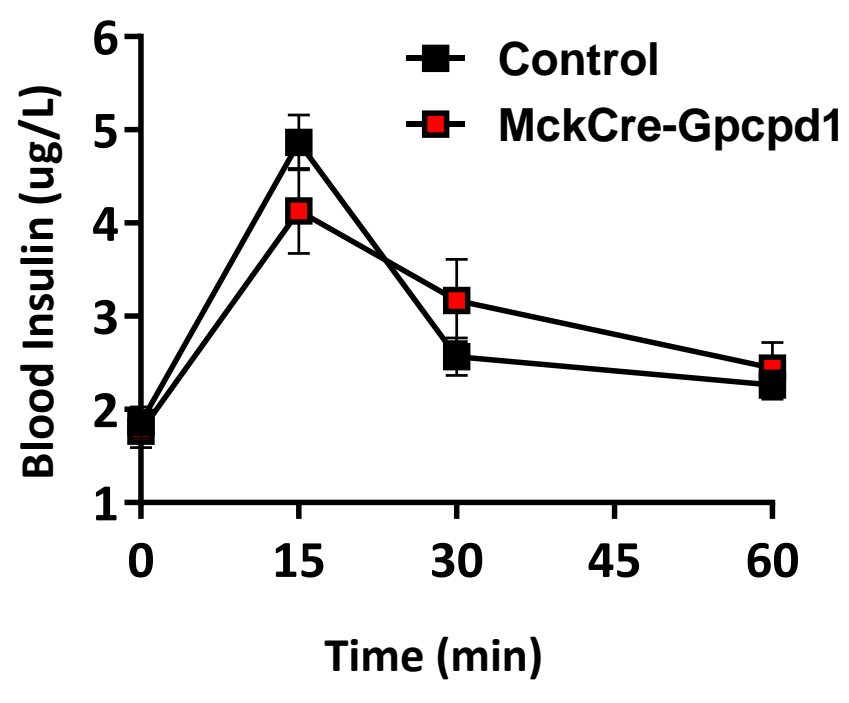




\section{Figure 4}

A

a Control

B

$\Delta \quad$ MckCre-Gpcpd1 a Control

$\triangle$ MckCre-Gpcpd1
C

- 6 months old

$\triangle 24$ months old
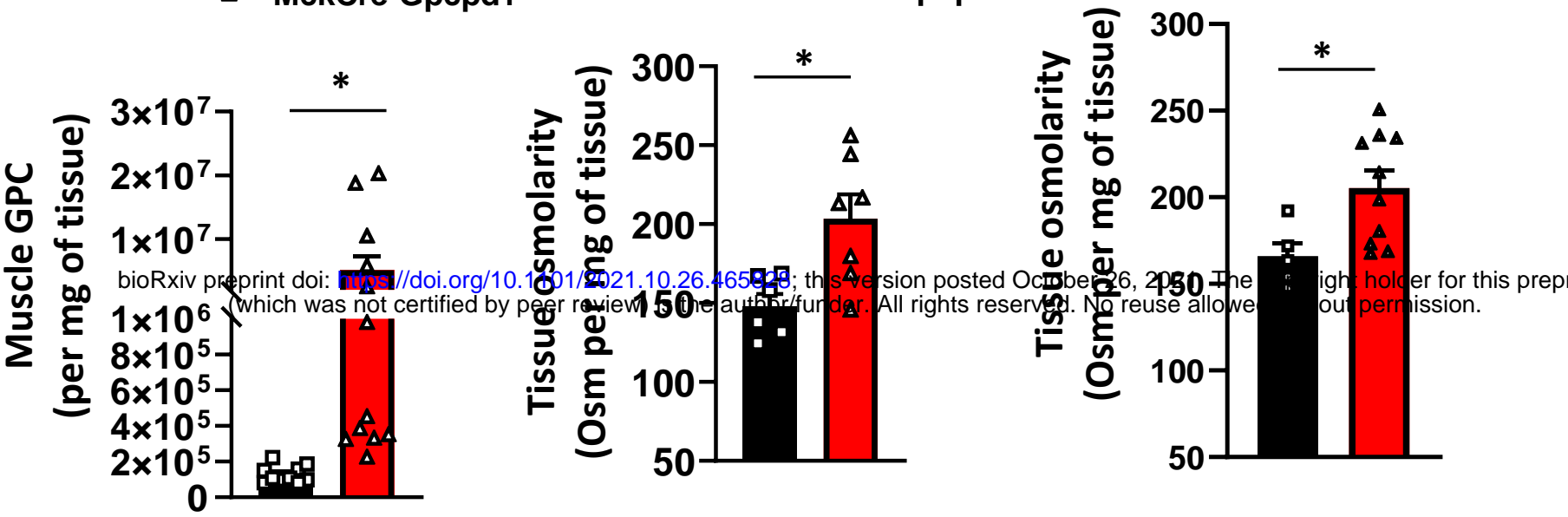

D

Mck-Gpcpd1 Control

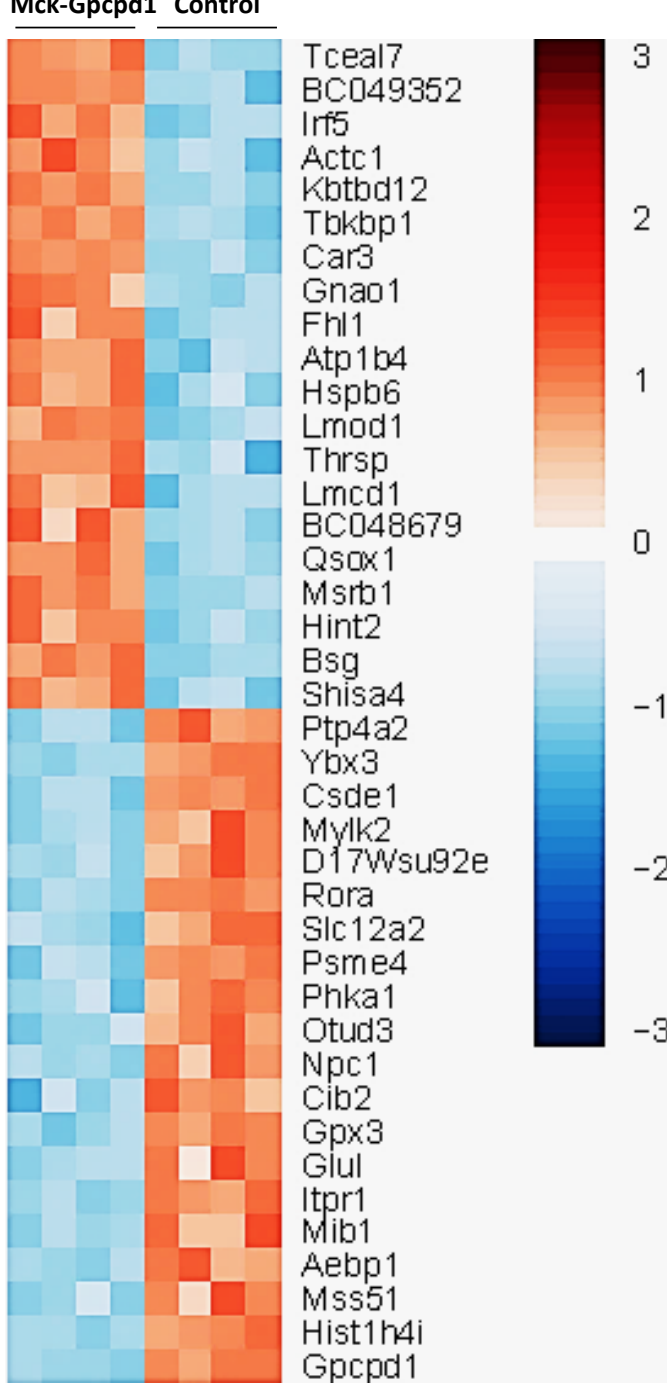

$\mathbf{F}$

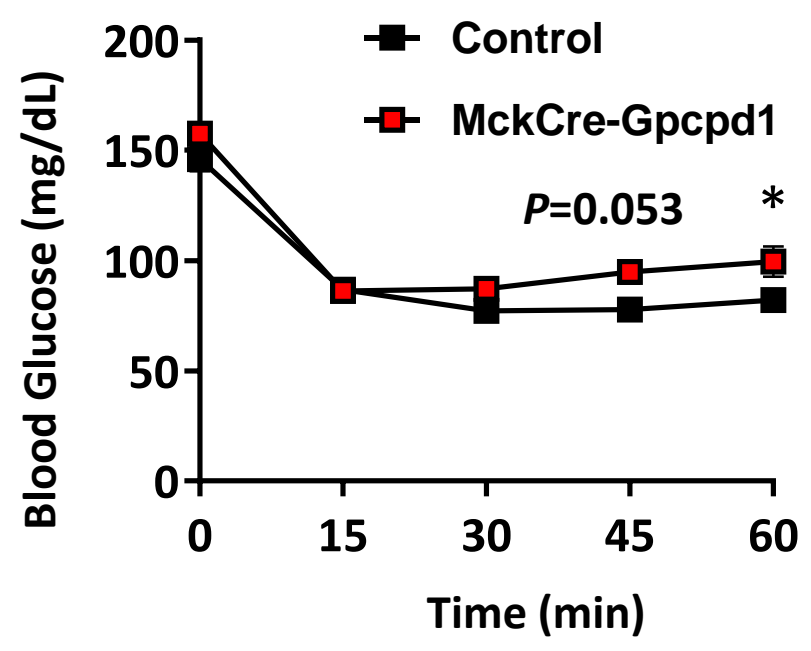

E positive regulation of catabolic process:1 glycogen metabolic process:3 glucan metabolic process: 5 cellular glucan metabolic process: 4 cellular carbohydrate metabolic process:2 positive regulation of cellular cataboli:6

insulin receptor signaling pathway:9 cellular response to peptide:10

cellular response to insulin stimulus:8 cellular response to hormone stimulus:7 cellular response to peptide hormone sti:11

energy reserve metabolic process:12

positive regulation of autophagy: 14 glucose metabolic process:15 cellular polysaccharide metabolic proces:13 response to insulin:16 transforming growth factor beta receptor:17 muscle system process:18 macroautophagy: 19 autophagy:20

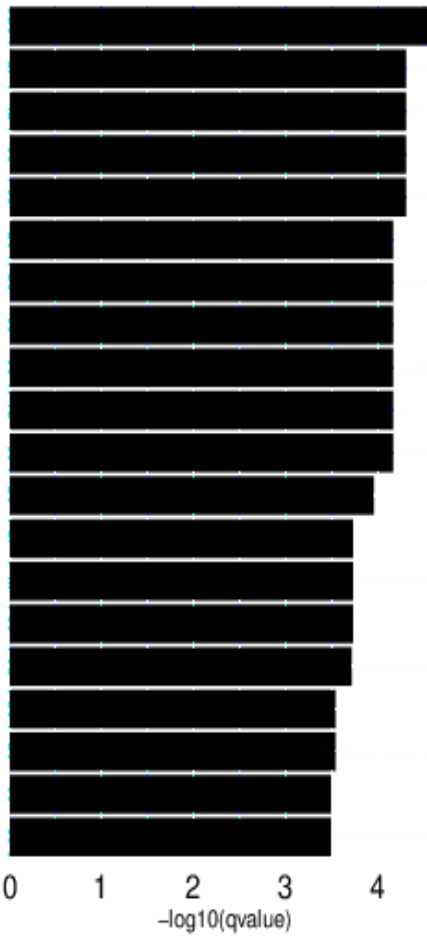

$\mathbf{G}$

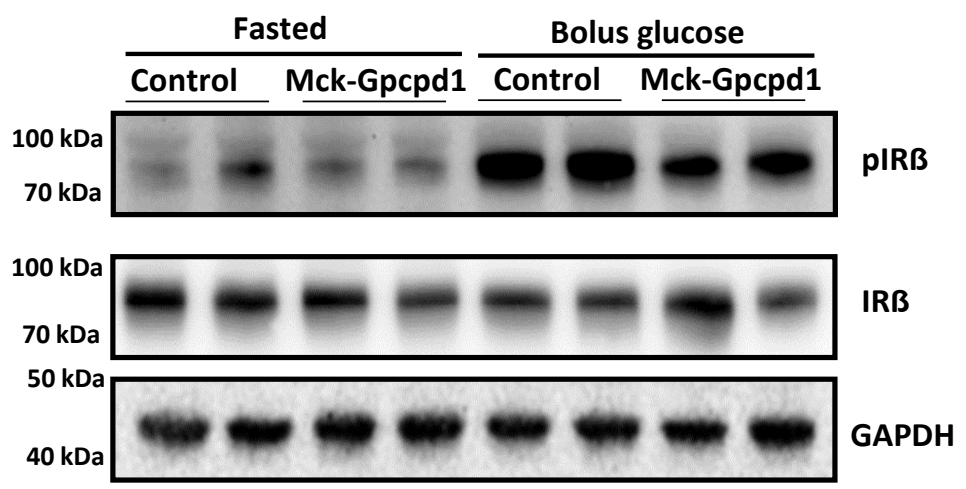


Figure 5

A

B
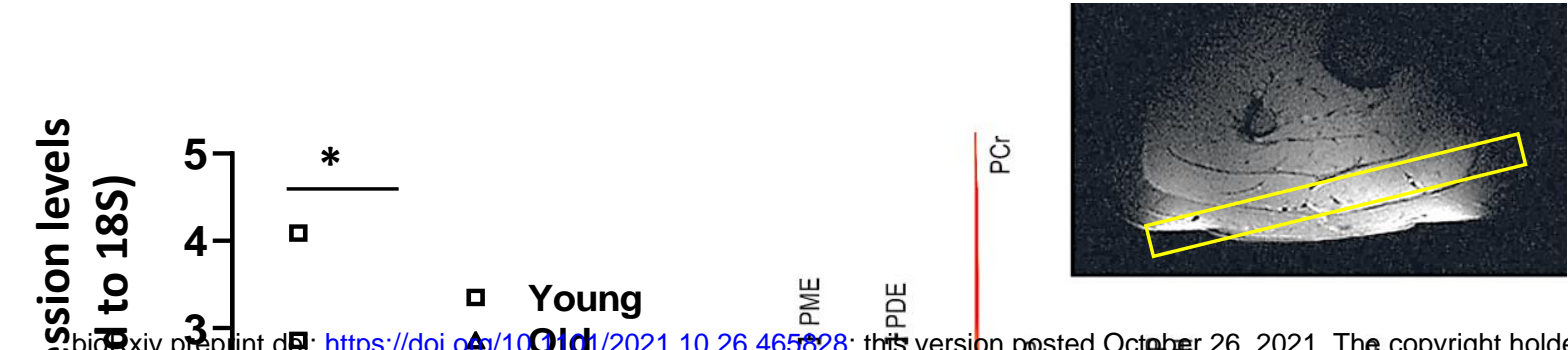

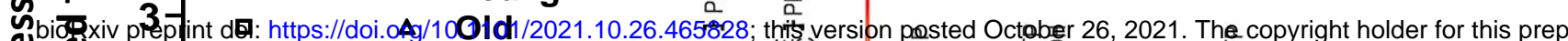
(which was not certified by peer review) is the author/fuudider. All righ

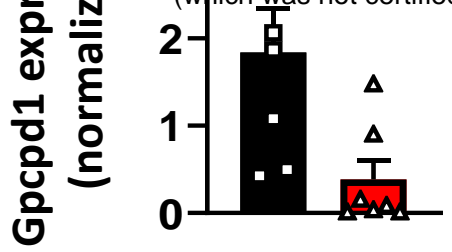

C

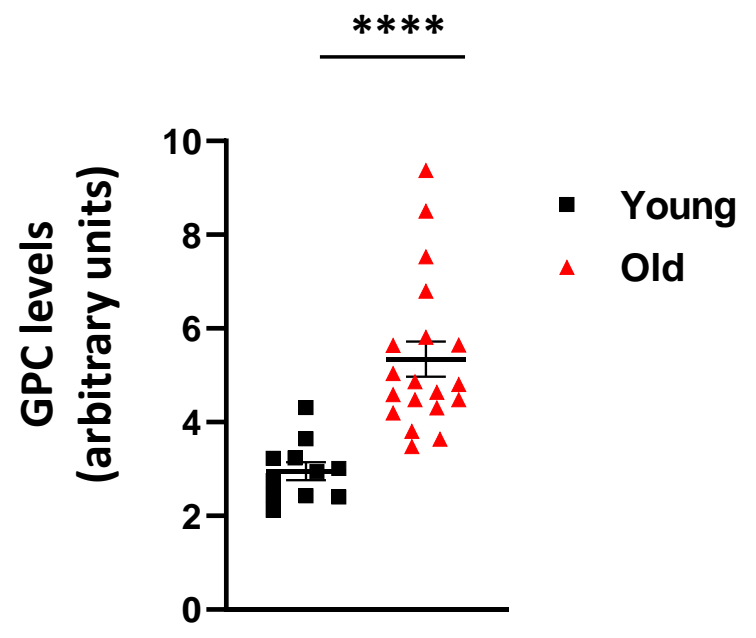

$\mathbf{E}$

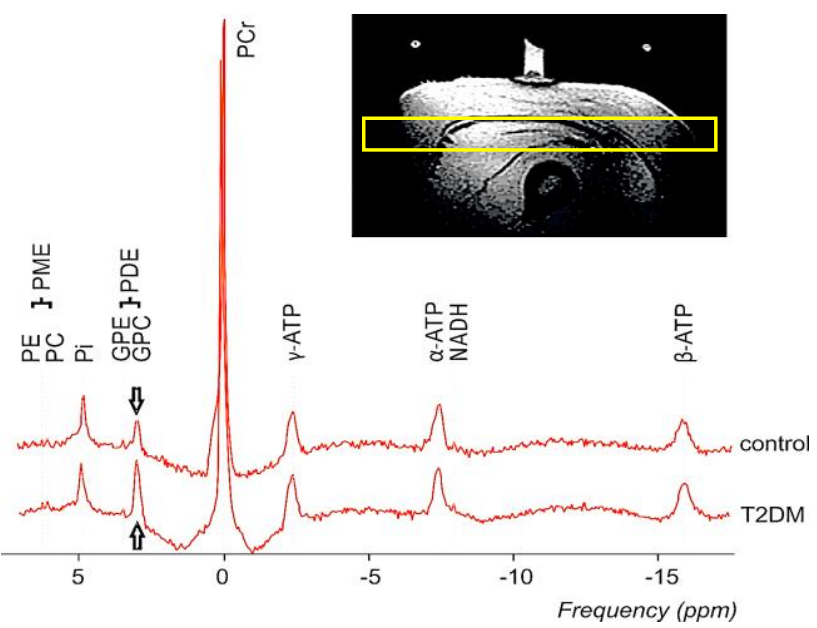

$\mathbf{F}$

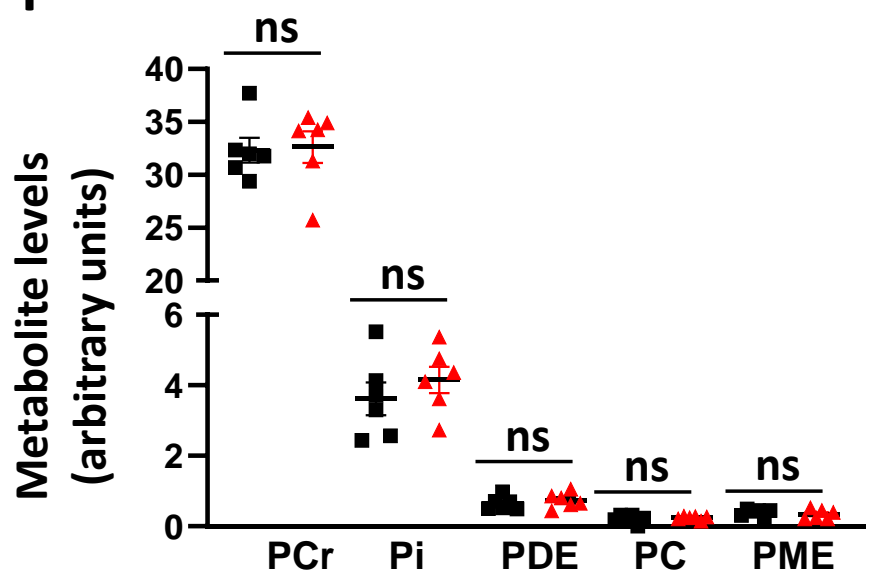

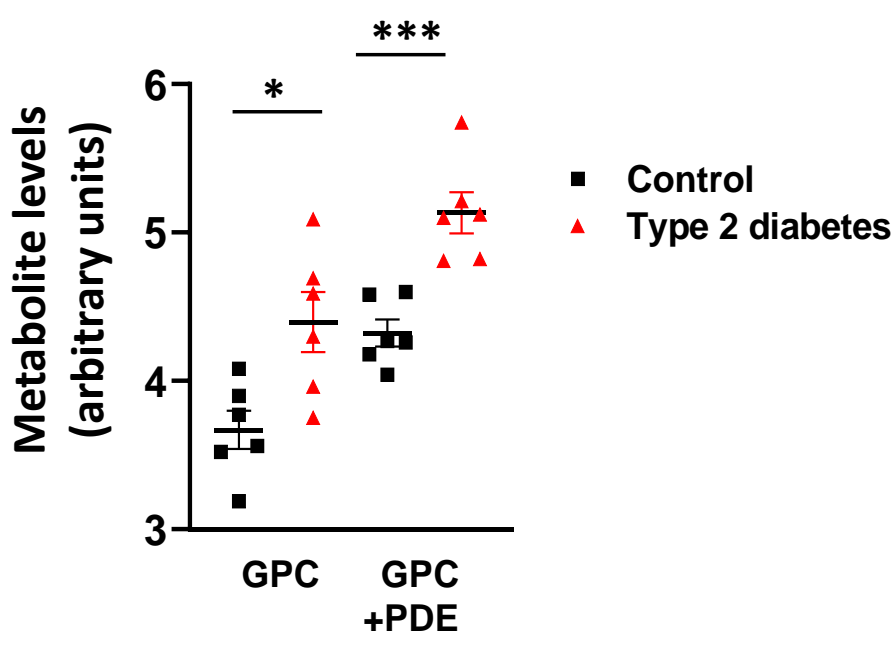

- Control

4 Type 2 diabetes 


\section{Supplementary Figure 1}
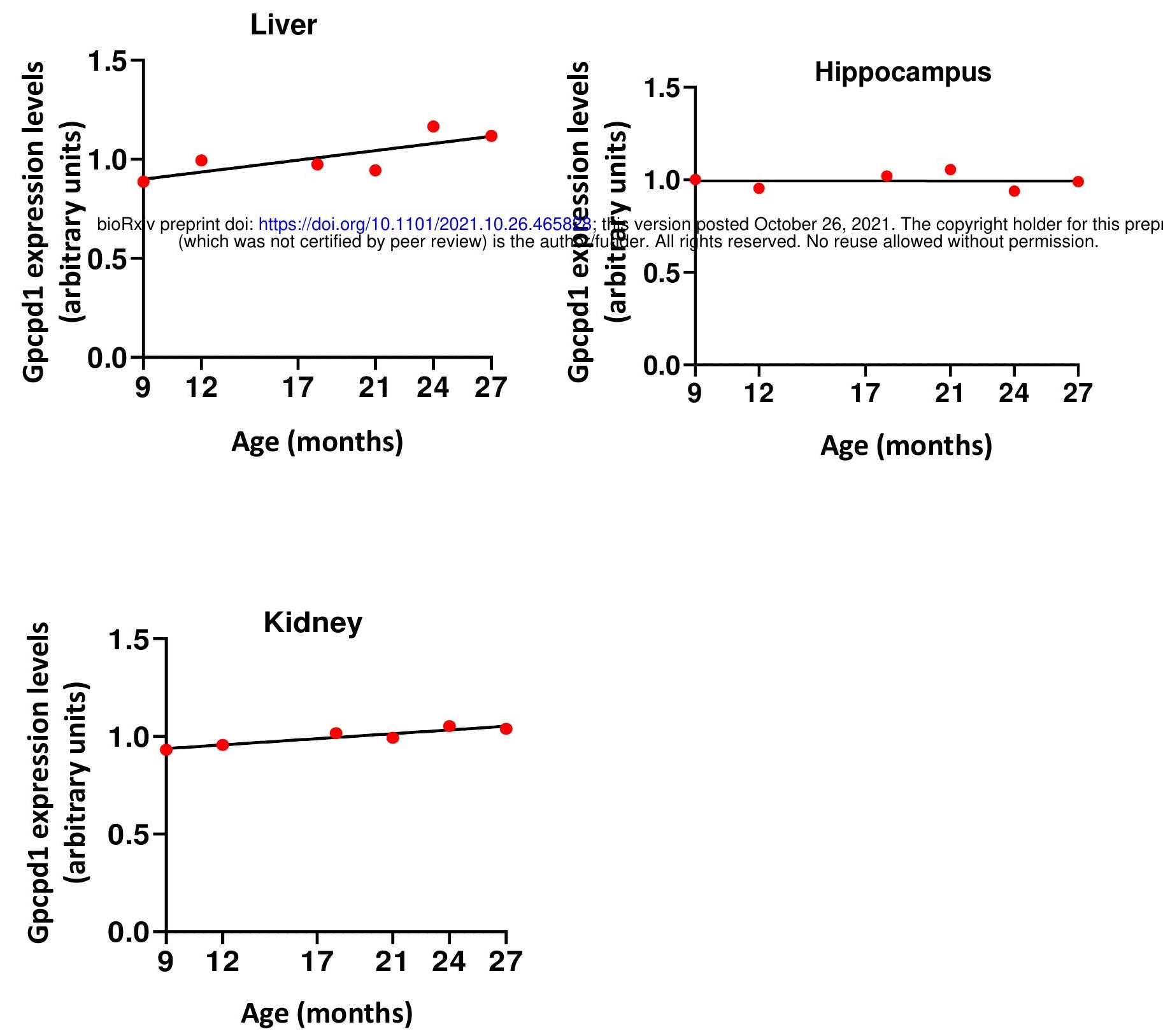


\section{Supplementary Figure 2}

A

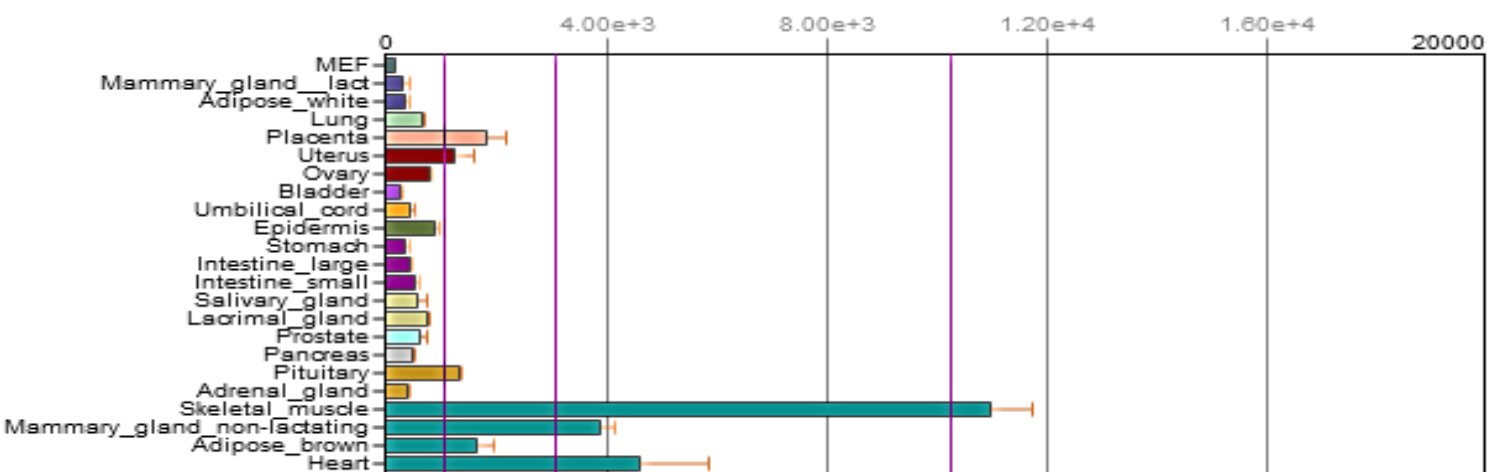

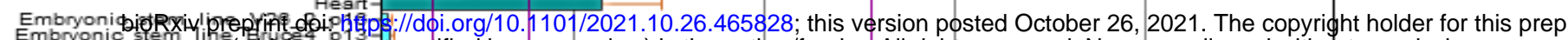
Emonic_ser

B

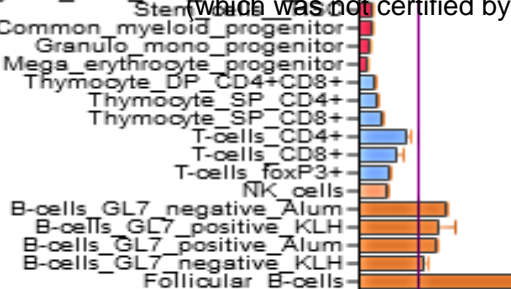

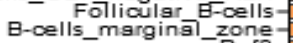

Mast $\infty$ lls-B

Mast_cells_lg Mast colls_l'sE

Dendritícolls myeloid CD8s-

Mendritic plasmsoytoid B220+-

Macos
Macro

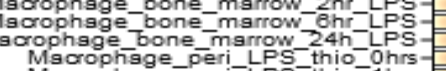

Macrophage-peri-LPS -thio-1 hrs =

Osteoblast_day $14=$

Nitions

$12=$

Neuro $29-1$

RAW_ ${ }^{264}$ Miñ $^{7}=$

Kidney

Cerebral_cortex Corefronts

Sping bulb

Hypothalamus

Nucleus_acoumbens-
Dorsalstriatum
Dorsal_root_ganglis-

Mesn(1023.3) 3xM

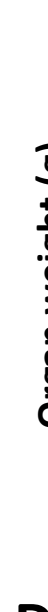

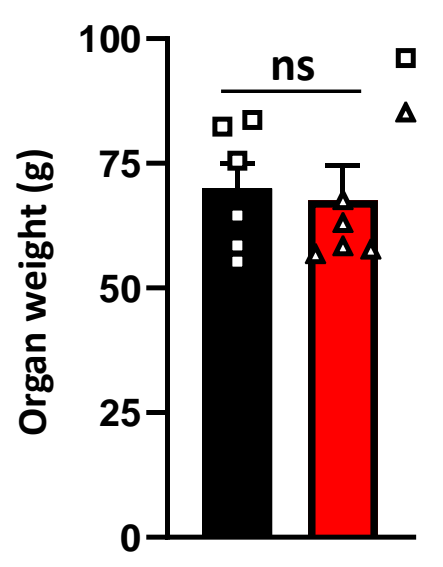

D

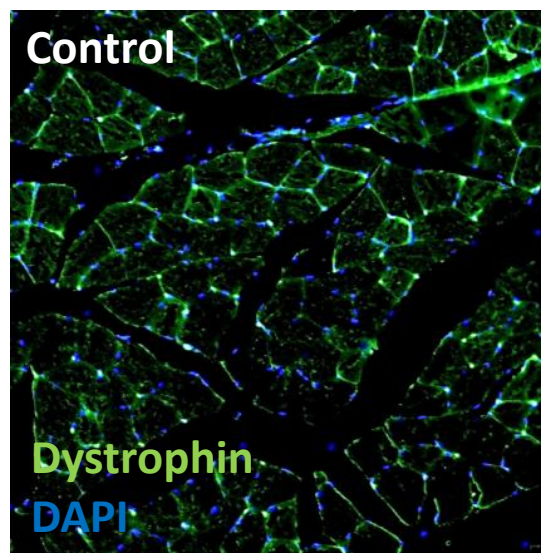

C

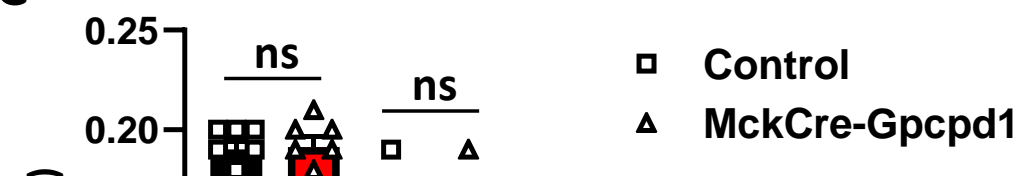

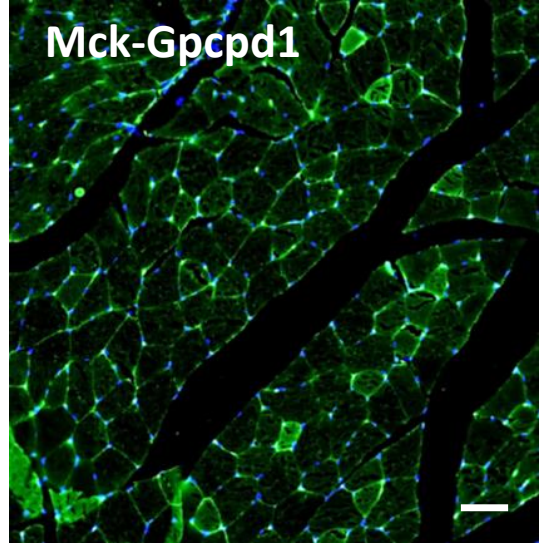

E Control

- MckCre-Gpcpd1

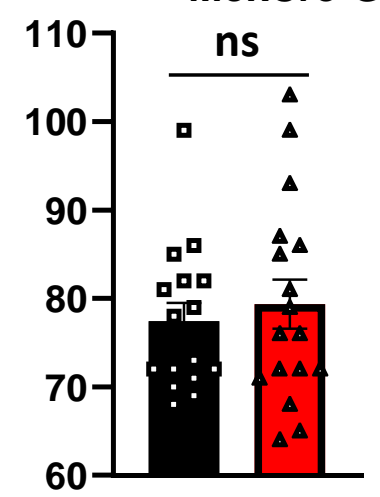




\section{Supplementary Figure 3}

A

- Control

$\Delta$ MckCre-Gpcpd1

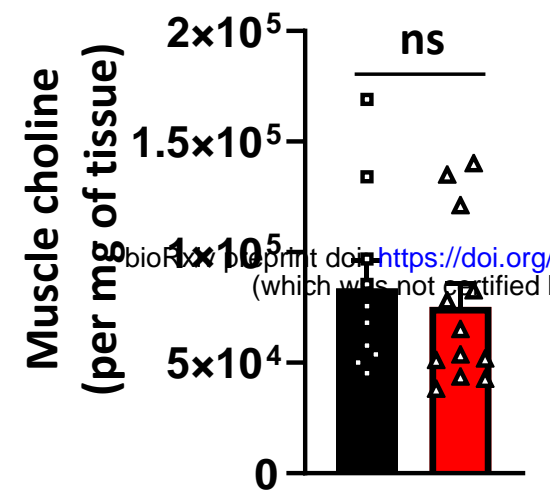

B

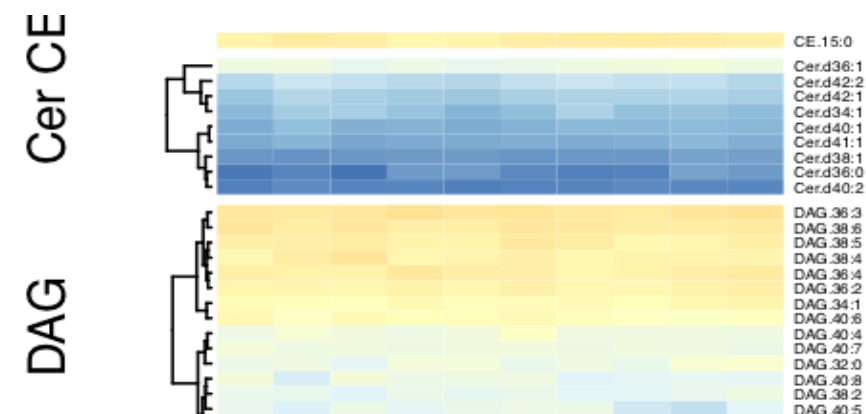

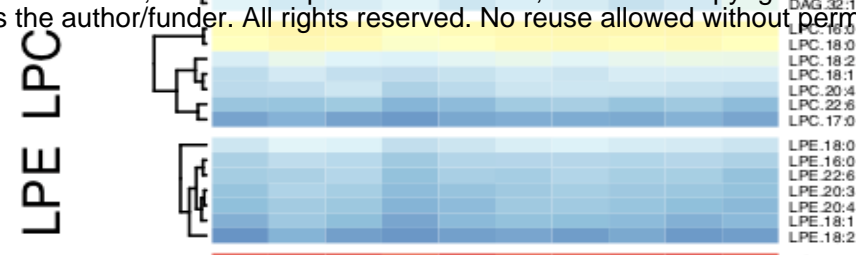

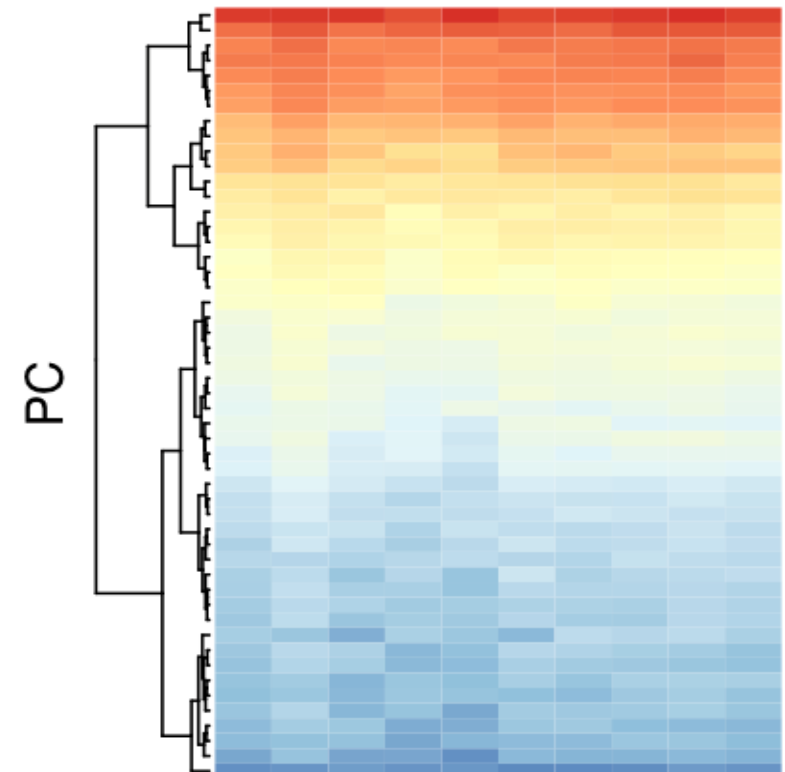

$\log (x+1$

$-10$

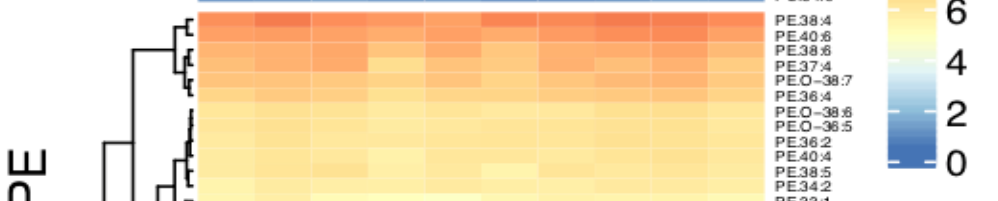

山

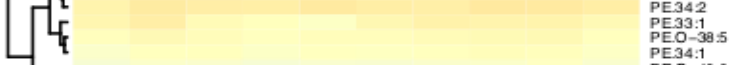

8

$\bar{n}$
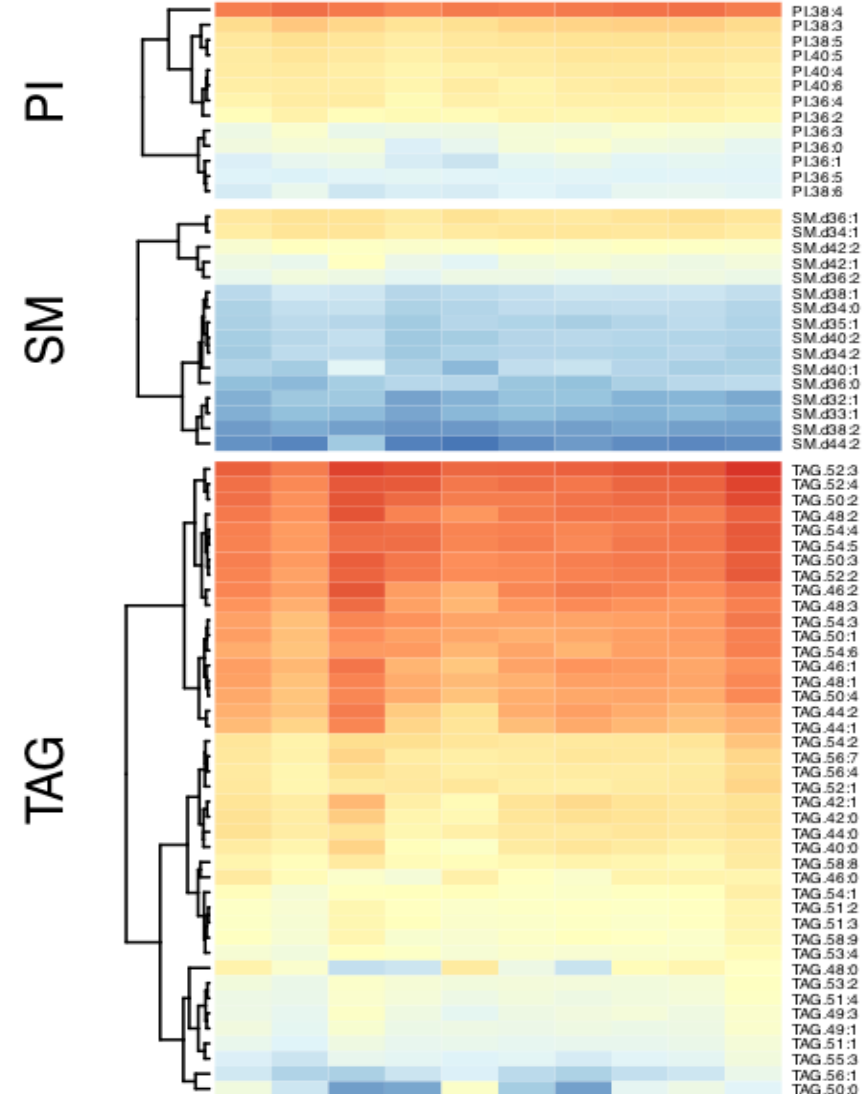

$\omega$ ம

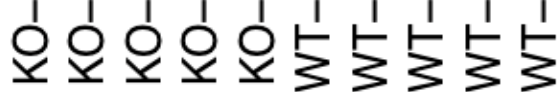
Mck-Gpcpd1 Control 


\section{Supplementary Figure 4}

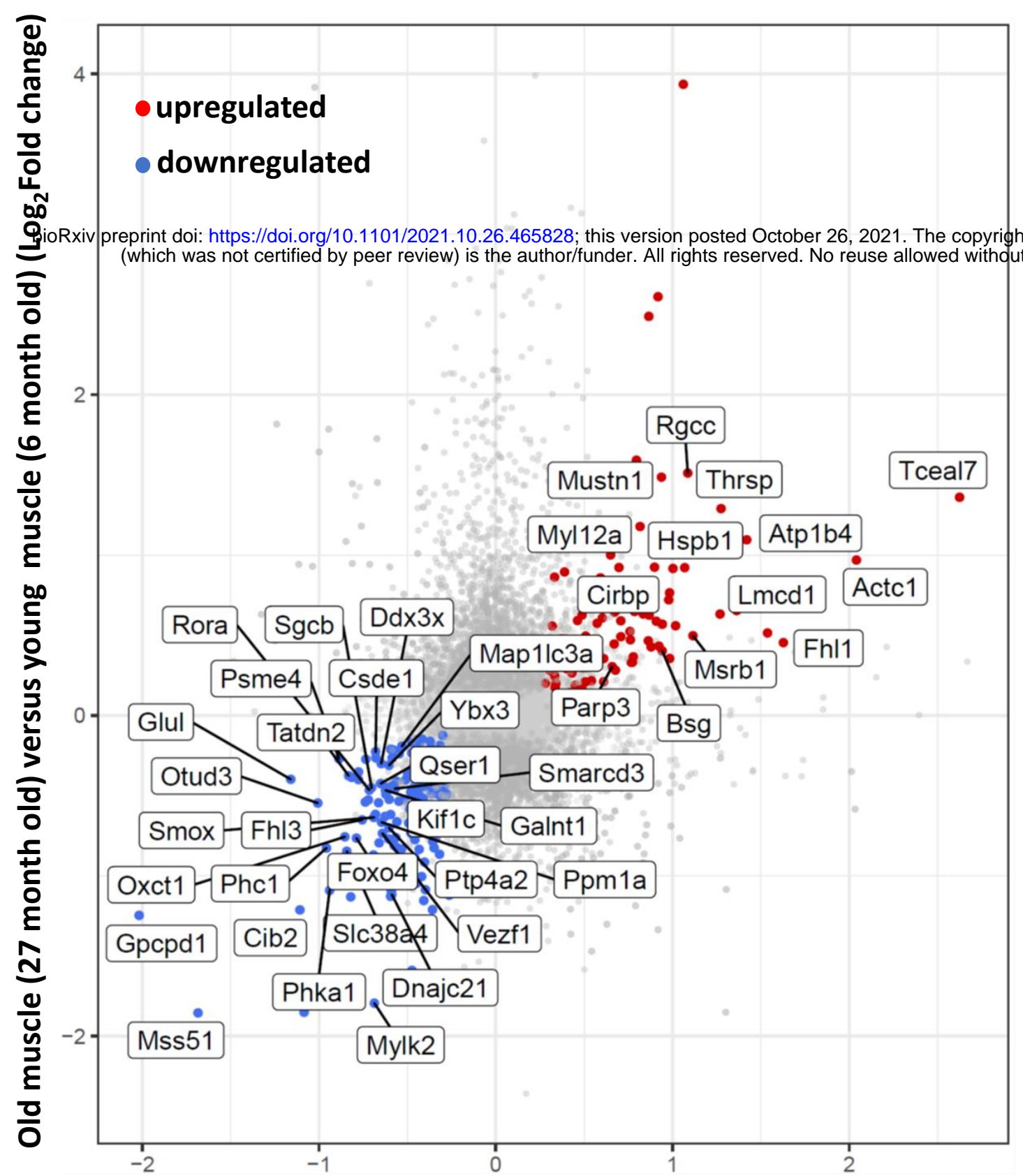

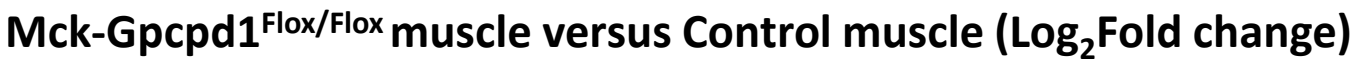

